\title{
Index for Volume 105
}

AUTHOR AND SUBJECT INDEX. Page numbers of errata are in italic.

Abad, J., S4.6, S4.24

Abad, Z. G., S4.15

Abawi, G. S., S1.5

Abbas, H. K., S4.1

Abbott, C. P., S4.1

Abdalla, O. A., S4.7

Abdelsamad, N., S4.1

Abdullah, S., S4.2

Abernethy, T., S4.6

Ables, G. W., S4.2

Aboughanem-Sabanadzovic, N., S2.10, S4.2, S4.3

Aboukhaddour, R., S4.3

Abrahamian, M., S4.67

Abreu, D. D. S., S4.3

Abucheli, G., S4.155

Accinelli, C., S4.1

Acevedo, M., 707, S4.160

Acharya, B., S4.49

Acharya, K., S4.22

Achor, D., 564

Adams, C. T., S4.178

Adaskaveg, J. E., 1302, S4.3, S4.52, S4.55, S4.57, S4.101, S4.168

Ades, P. K., 358

Adhab, M. A., S4.3

Adhikari, T., S4.3

Adkins, J. I., S4.4

Adkins, S., 388, S4.11

Adkison, H., S2.10, S4.141

Aegerter, B., S4.111

Affia, H., S4.4

Affokpon, A., 350

Aflatoxin: assessment of contamination of maize

in western Kenya, 1250

Afolabi, A. R., S4.4

Afolabi, C. G., S4.4

Agbetiameh, D., S4.63

Agnew, M., S4.104

Agrobacterium tumefaciens: gene transfer review article, 1288; overexpression of HspL promotes virulence in Arabidopsis, 160

Agudelo, P., 674, S4.23, S4.81

Aguilar, C. G., S4.4

Aguirre-Rayo, J. M., S4.128

Aguirre-Rayo, P., S4.128

Ah Fong, A., S4.67

Ahmed, H. U., S4.26, S4.62

Ahonsi, M., S4.49

Ahsan, M., S4.117

Ailloud, F., S4.162

Aitken, E. A., S4.137

Ajayi, M. A., S4.104

Ajayi, O. O., S4.4, S4.90

Akello, J., S4.68

Akino, S., S4.58

Akinsanmi, F., S4.4

Alabi, O. J., S4.5, S4.27, S4.98

Alam, M. W., S4.117

Alananbeh, K. M., 990

Al-Daoud, F., S4.52, S4.92

Alderman, S., S4.70, S4.124

Alexander, M., S4.34, S4.97

Alexandrov, N., S4.138

Alfenas, A. C., 1229, S4.44

Alford, B., S4.5

Alger, E. I., S4.5

Alhashel, A. F., S4.6

Ali, A., S4.6, S4.39

Ali, G. S., 1183, S4.6, S4.71

Ali, J. G., S4.164

Ali, M., 1183, S4.6
Ali, S., 482, S4.2

Ali, S. A., S4.40

Aliferis, K. A., 1334, S4.130

Alignment-guided mutagenesis: of an attenuated, cross-protective Pepino mosaic virus variant, 126 Alioto, D., 135

Alkharouf, N., S4.142

Allan, E., S1.5, S4.6

Allen, C., S4.6, S4.72, S4.85, S4.138, S4.139, S4.162

Allen, T., S2.11, S4.20, S4.130, S4.148, S4.181

Allen, T. W., S2.10, S2.12, S4.3, S4.68, S4.88

Allen, W. C., S3.3, S4.126

Allium spp.

-A. cepa: genetic and pathogenic variability of

Fusarium oxysporum f. sp. cepae in, 525

-A. fistulosum: genetic and pathogenic variability

of Fusarium oxysporum f. sp. cepae in, 525

Al-Mahmooli, I. H. S., 1229

Almeida, M. R. A., 805

Almeida, R., S4.171

Almeyda, C. V., S4.6

Almond leaf scorch disease: Xylella fastidiosa associated, population structure, 825

Alpizar, L. G., S4.27

Alruwaili, H., S4.29

Al Rwahnih, M., 758, 840, S4.5, S4.11

Al-Sadi, A. M., 1229

Al-Saleh, M. A., S4.7

Alshahwan, I. M., S4.7

Altenbach, D., S4.34

Alternaria spp.: associated with fruit rot of blueberry

in California, characterization of, 1555

Alvarez, E., S1.1, S1.3, S1.5, S1.6, S4.47, S4.180

Amaradasa, B. S., S4.7, S4.41

Amarasinghe, C., S4.43

Amer, M. A., S4.7

Ames, K. A., S4.16

Amiri, A., S4.7, S4.45, S4.160

Ampelomyces quisqualis: transcriptional reprogramming during host-induced germination, 199

Amsden, B., S2.7, S4.146

Amyotte, S., S4.176

Analytical and theoretical plant pathology: improved predictive performance of combined models, 1174; information graphs for binary predictors, 9;

Sr36- and Sr5-mediated resistance response to Puccinia graminis f. sp. tritici associated with guard cell callose deposition, 728; testing the independent action hypothesis of pathogen mode of action, 18; tolerance to 'Candidatus Liberibacter solanacearum' in potato germplasm, 1573

Anchieta, A., S4.77, S4.82, S4.133, S4.176

Anco, D., S4.7

Ancona, V., S4.27

Andersen, K. F., 210

Anderson, C. T., S4.88

Anderson, D., S4.62

Anderson, G., S4.115

Anderson, J. A., 656

Anderson, M. M., S4.134

Anderson, W., 1522

Anderson Paul, P., S4.33

Andrade-Piedra, J., S4.59, S4.141

Andreason, S., S4.124

Andrivon, D., S4.88

Androdias, A., S4.88

Angel, C. A., S4.3, S4.8, S4.23, S4.157

Angel, J. C., S4.8, S4.23

Anger, N., S4.129
Anh, N. T., 1568

Anh, V. L., 1568

Antioxidative system: in soybean leaves, changes with infection by Corynespora cassiicola, 1050 Antonise, R., 1220

Aouini, L., S4.8

Appel, D. N., S2.1, S2.7

Apricot ringpox disease, 399

Ara, J., S4.10, S4.40, S4.102

Arabiat, S., S4.71

Arabidopsis thaliana: overexpression of $\mathrm{HspL}$ promotes

Agrobacterium tumefaciens virulence in, 160 Araldi da Silva, G., S4.8

Aranda, M. A., 1389

Araújo, L., S4.23, S4.118

Arcibal, E., S4.180

Arciniega, A., S4.14

Arellano, C., S2.7

Arias, O., S4.109

Arias, R., S4.89

Arif, M., S4.8, S4.22, S4.131

Arismendi, N. L., S4.98

Aristizabal, N., S1.1

Arne, S., S4.9

Arneson, N. J., S4.99

Arnold, K., S4.8

Arone, L. R., S4.8

Arpaci, B. B., S4.22

Arpaia, M. L., S4.92

Arseneault, T., 1311

Artemisia absinthium, 1183

Artichoke latent virus: complete nucleotide

sequence places it in Macluravirus, 1155

Aruppillai, S., S4.9

Asalf, B., S4.9

Asano, S., S4.9

Asea, G., 956

Ashiglar, S., S4.16, S4.132

Atehnkeng, J., S4.63

Atha, B., S4.73

Atha, B. W., S3.1

Athey, J., S4.33

Atibalentja, N., S4.9

Aucique-Pérez, C. E., S4.23, S4.34

Augusto, J., S4.63

Auguy, F., S4.37

Aujla, I. S., S4.9

Avena sativa: QTLs from genotypes conditioning resistance to Puccinia coronata, 239

Avenot, H. F., S4.10

Avila-Adame, C., S4.10

Avrova, A., S4.45

Ayliffe, M. A., 872

Ayo-John, E. I., S4.4

Ayyemperumal, J., S4.10

Azevedo, R. F., S4.50

Azole resistance: mechanisms in Erysiphe necator, 370

Babiker, E. M., 239

Babu, B., S2.8, S4.10, S4.67, S4.101

Backus, E. A., 608, S4.10

Bacon, C. W., S4.10

Bacterial spot resistance: association analysis in tomato, 1437

Bacteriology: 'Candidatus Liberibacter solanacearum' genome plasticity in haplotype A, 863; genetic diversity of Indian populations of 'Candidatus Liberibacter asiaticus', 1043; genetic diversity of New York populations of 
Clavibacter michiganensis subsp. michiganensis, 169; light influence on Pseudomonas cichorii JBC1 severity in tomato, 412; novel DSF-controlled virulence-associated genes in Xanthomonas oryzae pv. oryzidola Rs105, 588; overexpression of HspL promotes Agrobacterium tumefaciens virulence in Arabidopsis, 160; Ralstonia solanacearum race 3 biovar 2, phylogeographic study and screening for resistance against its core effectors, 597, cover photo: May; Ralstonia solanacearum type III effectors associated with virulence on tomato and eggplant, 1529; sharpshooter egestion and salivation of Xylella fastidiosa, 608; streptomycin resistance in California Erwinia amylovora isolates, $1302 ;$ Xylella fastidiosa isolates from subsp. multiplex and fastidiosa cause disease on southern highbush blueberry, 855

Badar, R., S4.10

Badillo-Vargas, I. E., S4.11

Bag, S., 840, S4.11, S4.134

Bagewadi, B., S4.143

Bagi, F., S4.21

Bahder, B. W., S4.134

Bai, Y., S4.173

Bais, H. P., S3.2

Bak, A., S4.15

Baker, C., S4.10

Baker, C. A., S4.11

Baker, H. N., S3.1, S3.3, S3.4, S4.180

Bakker, P. A. H., S4.147

Bakkeren, G., 728, S4.107

Balatti, P., S4.45, S4.46

Balci, Y., S1.1, S3.1

Balesdent, M. H., S4.176

Bampi, D., S4.11

Bandara, A. Y., S4.11, S4.12

Banderas, J., S4.12

Bandyopadhyay, R., S4.63, S4.68

Bansal, A., S4.12

Bao, M., S4.175

Barak, J. D., S4.39

Barandoc-Alviar, K., S4.12, S4.89

Barash, I., S4.49

Barba, P., 1097

Bardsley, S., S4.12

Barnier, J., S4.154

Barocco, R. L., S2.1

Barone, M., 135

Barphagha, I. K., S4.109

Bartels, M., 1496

Bartz, J. A., S4.40

Basent, B. R., 872

Bashir, H., S4.117

Basil downy mildew resistance: in Ocimum species, 778

Basnet, B. R., 470

Batista, J. A. N., 628

Batista da Silva, M., S4.13

Batson, A., S4.20

Batuman, O., S4.13, S4.28

Batzer, J. C., S4.59

Bauchan, G., S4.75

Bauchan, G. R., 91, 1013, S4.121

Baucom, D., S4.13

Baudoin, A., S4.10

Baufume, S., S4.37

Baum, T. J., 1362, S4.111

Baumgartner, K., 255, S4.13, S4.47, S4.78, S4.138

Baur, M., S4.42

Bayasal-Gurel, F., S4.13, S4.14

Bazor, B. A., S4.28

$b c-3$ resistance allele, 1487

Beach, S., S4.14

Beale, J., S4.146

Bean common mosaic virus: isolate able to overcome the $b c-3$ resistance allele in common bean, 1487

Beard, J. J., 1013, S4.121

Beaudoin, E., S1.6
Beaulieu, J. R., S3.1

Beaver, J., S1.2, S1.4

Becker, C. M., S4.178

Beckerman, J. L., S4.1

Beckham, K., S2.8

Beed, F., S4.59, S4.127

Beede, R., S4.52

Beeman, A., S4.14

Beerepoot, A., S4.149

Behringer, G., S3.2

Beirn, L. A., S3.2

Bekhiet, M. A., S4.138

Bekoscke, K. A., S4.176

Belasque, J., Jr., S4.51

Belfield, K. D., 1183

Beligala, G. U., S4.14

Belisario, A., 1427

Bell, A. A., S4.31, S4.84, S4.94

Bellaloui, N., S4.94

Bellin, D., 199

Bellizzi, M., S4.95

Bemisia tabaci: interactions with cassava brown streak disease, 646

Ben-Dov, Y., S4.22

Benitez, E. R., S4.14

Benjain, T., S4.158

Ben-Naim, Y., 778, 1446

Bennett, R., S4.132

Berendsen, S., S4.14

Berepiki, A., S4.45

Bergamin Filho, A., S4.87

Bergstrom, G. C., 441, 695

Bermúdez-Cardona, M. B., 26

Bernardeli, A. M. A., 1050

Bernardy, M., S4.151

Berner, D. K., 91

Bernhardt, E. A., S4.164, S4.165

Beruga, M., S4.15

Best, G. S., S4.15, S4.45

Bester, G., 1573

Betaflexiviridae: possible new genus within, 399

Betancourth, C. A., S1.6

Beucke, K. A., S4.71

Bevels, E., S4.33

Beyl, C. A., S4.55

Bhagwat, B., S4.151

Bharathan, S., S4.100

Bhat, R., S4.52

Bhatnagar, D., S4.117, S4.134

Bhavani, S., 470, 477, 872

Bhose, S., 1043

Bian, Z., S4.15

Bianchedi, P., 555

Bianco, T., S4.145

Bienapfl, J. C., S4.15

Bigott, A. F., S4.16

Bill, B. S., S4.68

Bilodeau, G., S4.96

Binary predictors: information graphs for, 9

Biochar. See Maple bark biochar

Biochemistry and cell biology: antioxidant system changes in soybean leaves infected by Corynespora cassiicola, 1050; effect of flumorph on F-actin dynamics in Phytophthora infestans, 419; effects of Monographella albescens infection on leaf gas exchange and chlorophyll $a$ fluorescence in rice, 180, cover photo: February; effects of potato-pysllid-vectored 'Candidatus Liberibacter solanacearum' infection on potato leaf and stem physiology, 189; effects of silicon on gas exchange and oxidative metabolism in rice leaves infected by Pyricularia oryzae, 738; effects of Stenocarpella macrospora infection on leaf gas exchange and chlorophyll $a$ fluorescence in maize, 26; silicon-induced systemic defense responses in perennial ryegrass against Magnaporthe oryzae infection, 748

Biological control: effect of Pseudomonas fluorescens LBUM223 on potato yield and common scab symptoms, 1311; PilG regulation of twitching motility and antifungal antibiotic biosynthesis in Lysobacter enzymogenes, 1318; transcriptional reprogramming of Ampelomyces quisqualis during host-induced germination, 199; Trichoderma sp. Tri-1 control of Sclerotinia sclerotiorum on oilseed rape, 1325

Biological indexing: versus next-generation sequencing for detection of grapevine viral pathogens, 758

Birch, J., S4.100

Birch, P., S4.45, S4.80, S4.97

Birch, P. R. J., 966

Biscaro, A., S4.139

Bishnoi, U., S3.2

Bispo, W. M. S., S4.23

Bissett, A., S4.131

Bissonnette, K. M., S4.16

Black, A., S4.129

Black, B., S4.16

Blackburn, N. J., S4.20

Blacutt, A., S4.16

Blakeslee, J. J., 126

Blanc, J., S3.1, S4.43

Blanche, S., S2.4

Blodgett, J. T., S4.16

Blomquist, C. L., S4.43, S4.119, S4.164, S4.165

Bloom, D. C., S4.15

Bloomingdale, C., S4.16

Bluhm, B., S4.42, S4.156

Blumeria graminis f. sp. tritici: direction

observation of ascosporic infection, 797, cover photo: June

Boateng, K., 69

Boberg, J., 1191

Bock, C. H., S4.17, S4.27, S4.28, S4.40, S4.51

Bockus, W., S4.32

Bocsanczy, A. M. B., S4.17

Bodaghi, S., S4.33, S4.135

Boenisch, M., S4.163

Bohannon, S., S4.158

Boissonnier, B., S4.79

Bokati, D., S4.17

Bokhari, S. S., S4.40

Bolton, M., S4.15, S4.58

Bolton, S. L., S4.17

Bomberger, R. A., S4.18

Bomme, G., S4.59

Bonato, A. L. V., 284

Bond, J., S4.63, S4.146

Bond, M., S4.33

Bonde, M. R., 91, S3.3, S4.18

Bondge, D., S4.162

Bonkoungou, S., S4.63

Bonman, J. M., 239

Borel, F. C., S4.18

Borges, A. V., S4.18

Borhan, H., S4.43

Borneman, J., S4.50, S4.154, S4.172

Borodynko, N., 1270

Borowicz, P., 707, S4.160

Borron, S., 1408

Borth, W., S4.80, S4.157

Bortolin, E., 1427

Bos, J., S4.80

Bosque-Pérez, N., S4.139

Bossa-Castro, A., S4.18

Bostock, R. M., S4.119, S4.121, S4.125

Botrytis cinerea: emergence of fungicide resistance in, 424; environmental conditions affect infection of grape berries more than strain or transposon genotype, 1090; factors affecting infection on grapevine inflorescences and young berry clusters, 325; in sweet basil, effect of

shoot potassium concentrations on, 1059

Boule, J., S4.112, S4.140

Bounds, R., S4.56, S4.178

Bourras, S., 1288

Bourret, T. B., S4.19 
Bouthillier, M., S4.151

Bouwnmeester, H., 646

Bova, L. P., 518

Bowden, R., S4.47

Bowden, R. L., S4.55, S4.68

Bowen, K., S2.4, S4.19

Bowen, P., 1373

Boyd, N., S2.6

Brachypodium distachyon-Cochliobolus sativus pathosystem, 482, cover photo: April

Bradeen, J. M., 1131, S4.141

Bradley, C. A., 1080, S4.4, S4.16, S4.30, S4.69,

S4.90, S4.126, S4.173

Bradshaw, T., S4.24

Bragard, C., S4.87

Branch, W. D., S2.6

Brannen, P., S4.19

Brannen, P. M., S4.17, S4.102

Brantner, J. R., S4.19, S4.26

Brassica napus: Trichoderma sp. Tri-1 control of Sclerotinia sclerotiorum on, 1325

Brassica seed meal soil amendments: effect on apple production, 460

Braun-Kiewnick, A., 1245

Brazee, N., S4.154

Brazee, N. J., S1.6

Bremia lactucae: resistance in lettuce ' $\mathrm{La}$

Brillante' conferred by Dm50 gene and multiple QTL, 1220

Brennan, M., 1183, S4.6

Brenneman, T. B., S2.1, S2.3, S2.11, S4.20

Brevipalpus spp., 1013

Brewer, M. T., 815, S4.35, S4.48, S4.82, S4.132, S4.134

Briggs, J., 1347, S4.121

Bright, D., S4.150

Brito, R. A., S4.25

Brlansky, R. H., 564, 1013, 1277, S1.3, S3.2, S4.57, S4.121

Brochard, N., S4.20

Broders, K., S1.6, S1.8, S4.99

Broders, K. D., S4.167, S4.177

Brodhagen, M., S4.20

Bronstein, P. A., S4.44

Broome, J. C., S4.78

Brown, A., S4.148

Brown, J., S4.12, S4.67

Brown, J. K., S4.7, S4.20, S4.159, S4.174

Brown, J. K. M., 895

Brown, K. A., S2.1, S4.20

Brown, R., S4.117

Brown, R. L., S4.134

Brown apical necrosis: environmental and pathogenic factors inducing infection on English walnut fruit, 1427

Browne, G., S4.52

Browne, G. T., S4.20, S4.37, S4.43

Brown-Guedira, G., 1123

Browning, K. S., S4.96

Broz, K., S4.163

Bruce, A., S4.20

Bruce, J., S4.97

Bruce, J. E., S4.34

Bruckhart, W. L., S4.21

Brueggeman, R., 500, 509, 707, S4.126, S4.160

Brueggeman, R. S., 99, S4.47, S4.74

Bryson, P. K., S4.61

Buchman, J. L., 1573

Buchner, R., S4.52

Buck, J., S2.8, S4.35

Buck, J. W., S2.3

Budakov, D., S4.21

Bulajić, A. R., S4.21, S4.76

Bull, C., S4.137

Bull, C. T., 316, S4.5, S4.101, S4.116

Bulluck, R., S4.166

Burbank, L., S4.21

Burch, K., S4.130

Burchhardt, K. M., 533
Burdman, S., S4.40

Burgin, L., 917

Burkhardt, A., S4.133

Burnett, F. J, 9

Burns, D., S2.4

Burr, T. J., S4.23, S4.57

Burris, E., S2.4

Burrows, M., S4.112

Burrows, M. E., S4.116

Bush, E., S3.3

Bushula, V., S4.21

Busot, G. Y., S4.8, S4.22, S4.96, S4.131

Butler, D., S4.60

Butler, D. M., S4.120, S4.127

Butler, S., S4.22

Butler, S. A., S2.2

Buzkan, N., S4.22

Byamukama, E., S4.22

Byrne, D., S4.67

Byrne, J. M., 378

Cabrera, J. A., S4.181

Caceres, M., S4.36, S4.84

Cacique, I. S., 738, S4.23

Cadavid, M., S4.8, S4.23

Cadet, R., S4.84

Cadle-Davidson, L., 370, 1097, 1104, S4.23

Caetano-Anolles, G., S4.123

Cai, R., 597

Caicedo, J. D., S1.4

Caicedo Chávez, J. D., S1.1

Callicott, K. A., S4.63

Campbell, A., S4.38

Campbell, H. L., S2.4

Campbell, L., S4.55

Campbell, P., S4.119

Canaday, C., S2.2

Cañas, S., S4.25

'Candidatus Liberibacter asiaticus': genetic diversity of Indian populations, 1043; incidence in citrandarins, 518

'Candidatus Liberibacter solanacearum': effects of potato-psyllid-vectored infection on potato leaf and stem physiology, 189; genome plasticity in haplotype A, 863; significance of late-season infections in management of, 929 ; tolerance to in potato germplasm, 1573

Candresse, T., 135, 1155

Canegallo, A., S4.23

Canik Orel, D., S4.23

Cannon, P. G., S4.132

Cano, L. M., S4.23, S4.24, S4.96

Cantoro, R., S4.23

Cantu, D., S4.47

Cao, J., S4.10

Cao, L., S4.175

Cao, M., S4.24

Cao, T., S4.51

Capouya, R. D., S4.24

Capsicum annuum: interactions of Phytophthora capsici with resistant and susceptible roots and stems, 1355; worldwide evaluation for Phytophthora fruit rot resistance, 110

Carbone, I., S4.137

Cardozo, L., S1.3

Carillo, D., S4.111

Carlson, M. O., S4.24

Carneiro, J. E. S., S4.80

Carneiro, R., 350

Carneiro, R. M. D. G., 628, 805

Caroline, J., S4.50

Carputo, D., 1131

Carr, J. P., S4.3

Carrillo, J., S4.80

Carrillo, J. D., S4.180

Carrillo-Ávila, E., S1.2

Carris, L. M., S4.123

Carroll, J., S4.24

Carson, M. L., 239
Carta, L., S4.25

Carter, A. H., S4.69

Carter, M., S4.25

Carvajal Yepes, M., S4.59

Cassava brown streak disease: interactions with

Bemisia tabaci, 646

Cassone, B., S4.144

Cassone, B. J., 956, S4.49

Castaneda, A., S4.71

Castella, G., S4.114

Castiblanco, L. F., S4.25

Castillo, J. A., 597

Castillo, P., S4.133

Castlebury, L. A., 990, S3.3, S4.35, S4.123, S4.126, S4.129

Castro, J. C., S4.25

Castroagudín, V. L., 284

Caswell-Chen, E. P., S4.122

Cavalcante, G. P., S4.25

Cecil, W., S4.133

Ceratocystis fimbriata: reassessment of recently described species on Mangifera indica, 1229

Cercospora zeae-maydis, 1080

Cereal root diseases: predicting with soil DNA and environmental parameters, 1069

Ceresini, P. C., 284, 1475

Cevallos, F., S4.25

Chagas Ferreira da Silva, E., S2.2, S2.11

Chalasani, N., S4.26

Chalivendra, S., S4.33

Chalupowicz, L., S4.49

Chamorro, M., S4.95

Chamorro Rodriguez, M., S4.26

Chan, B., S4.174

Chan, P. H., S4.26

Chanda, A. K., S2.2, S4.19, S4.26, S4.128

Chang, C. J., S4.133

Chang, H. X., S4.27

Chang, K. F., S4.26

Chang, P. F., S4.26

Chang, P. F. L., S4.83

Chang, T. D., S4.83

Chao, S., 239

Chao, S. M., 1206

Chapara, V. R., 1080

Charkowski, A. O., S4.6, S4.46, S4.121, S4.144, S4.179

Charlesworth, S. R., S4.65

Chase, C. D., S4.99

Chastagner, G. A., S4.38, S4.48, S4.49, S4.80, S4.93

Chaudhary, S., S4.27

Chauhan, R. P., S2.2

Chavan, S., S4.27

Chavarro Mesa, E., 1475

Chaverri, P., S4.129

Chaves, E., S4.27

Chavez, J., S4.34, S4.97

Chee, P. W., S4.13

Chen, A. Y., S4.27

Chen, C., S4.17, S4.27

Chen, C. C., S4.26

Chen, H., S2.2, S4.82

Chen, J., 189, 1573, S4.71, S4.133, S4.143, S4.159

Chen, K. S., S4.26

Chen, L., S4.111

Chen, L. F., S4.28

Chen, L.-F., S4.90, S4.143

Chen, P., S4.32, S4.73, S4.74

Chen, P. J., S4.150

Chen, S., 1347, S4.88, S4.154, S4.181

Chen, T. Y., S4.28

Chen, W., S4.3, S4.64, S4.70, S4.85, S4.152

Chen, X., 477, S4.69, S4.144, S4.151

Chen, X. M., 1206

Chen, Y., 1318, S4.28, S4.87

Chen, Y. J., S4.28, S4.107

Chen, Z., S2.5, S4.61, S4.117

Chen, Z. Y., S4.118 
Cheng, T.-F., 160

Cheon, W., S4.28, S4.79

Cherifi, F., S4.120

Cherry rusty mottle-associated viruses, 399

Cherry rusty mottle disease, 399

Cherry twisted leaf-associated virus, 399

Cherry twisted leaf disease, 399

Chewachong, G. M., 126

Chey, M., S4.100

Chi, F. L., S4.151

Chi, M. H., S4.17

Chiang, K. S., S4.28

Chikh-Ali, M., S4.29

Chikoti, P. C., S4.98

Childers, R., 342

Chilian, R. J., S4.53

Chilvers, M. I., 378, 1601, S4.34, S4.90, S4.102, S4.120, S4.146, S4.173

Chin, E., S4.97

Chingandu, N., S4.20

Chiroleu, F., 1529

Chitrampalam, P., S4.29

Chiu, Y. H., S4.83

Chlorophyll $a$ fluorescence: effects of Monographella

albescens infection in rice, 180; in maize, effects of

Stenocarpella macrospora infection on, 26

Cho, H., S4.29

Choi, G. S., S4.29, S4.155

Choi, S. K., S4.29, S4.155

Choi, Y. J., S4.77

Chou, H. P., S4.29

Choudhary, N., 564

Choudhury, R. A., S4.176

Chrysanthemum: systemic Puccinai horiana

infection, 91, cover photo: January

Chuma, I., 495, 1568

Chung, J., S4.56

Chung, K. R., S4.153

Chung, W. H., S4.30, S4.107, S4.154

Chuwa, C., 646

Ciancio, A., S4.163

Cilia, M., S4.34, S4.97

Ciliberti, N., 325, 1090

Citrandarins: incidence of 'Candidatus

Liberibacter asiaticus' in, 518

Citrullus spp.: inheritance of powdery mildew race 1W resistance, 1446, cover photo: November

-C. lanatus var. citroides, 1446, cover photo: November

Citrus leprosis virus: complex relationships between viruses, hosts, and vectors related to citrus leprosis disease, 1013; history and diversity recorded in herbarium specimens, 1277; nuclear type, identification and molecular characterization, 564

Citrus spp.: identification and molecular

characterization of nuclear Citrus leprosis virus, 564

Civerolo, E. L., 825

Claassen, B. J., S4.30

Clark, C. A., S4.158

Clark, K., S4.107

Clark, S., S4.30

Clarke, B. B., S3.2, S4.180

Clarke, C. R., 597, S4.93

Clavibacter michiganensis subsp. michiganensis: genetic diversity in New York populations, 169

Clay, B., S4.176

Clemente, G., 895

Clemente, T., S4.70

Clements, J., S1.6, S4.24

Cleveland, K., S4.37

Coaker, G., S4.12, S4.104

Coats, K. P., S4.49, S4.80

Cobb, R. C., S4.170

Cobine, P., S2.7

Cobine, P. A., 855

Coburn, A., S4.164
Cochliobolus sativus: Brachypodium distachyonCochliobolus sativus pathosystem, 482, cover photo: April

Cochran, A., S4.30

Cochran, A. M., S2.3

Cody, S., S4.152

Coelho, M., S4.50

Coffea canephora: resistance to Meloidogyne spp., 805

Coffee rust: review article, 1164, cover photo:

September

Coffey, M., S4.96

Coffey, M. D., 265, S4.145

Cohen, Y., 778, 998, 1446

Colagiero, M., S4.163

Collins, S. B., S4.30

Collyer, D., S4.114

Comstock, J. C., S4.43, S4.60

Concklin, M., S4.24

Conley, S. P., 1080, S4.126

Connacher, R., S4.100

Conner, K., S4.55

Conner, R. L., S4.26

Cook, D., S4.5, S4.13

Cooley, D., S4.24

Cooley, D. R., S1.6

Coons, K., S4.78

Coop, L. B., 35

Cooper, M., S4.8

Cooper, S., S4.164

Copes, W., S4.30

Copley, T. R., 1334

Corbière, R., S4.88

Cordova, L. G., S4.31, S4.104

Cornelius, L., S4.119

Correa, V. R., 805

Correll, J., S4.42, S4.84, S4.120

Correll, J. C., S4.109

Corynespora cassiicola: antioxidant system changes in soybean leaves infected by, 1050

Cosme, L., S4.81

Costa, R. V., S4.130

Costa de Novaes, M. I., S4.108

Cota, L. V., S4.130

Cottrell, T. E., S4.27

Cotty, P. J., S4.8, S4.41, S4.63, S4.64, S4.68, S4.128

Cowger, C., 1417, S2.7, S4.94

Cox, J. A., 917

Cox, K. D., S4.176

Coyne, D., 350, S4.59

Cr811 gene, 764

Cramner, T., S4.52

Crane, E. A., S4.19

Crane, J., S4.31

Cranmer, T., S4.92

Cranny, Z., S4.20

Crassweller, R., S4.24

Craven, K., S4.17

Creamer, R., S4.13, S4.103

Cregan, P., S4.108

Crenshaw, N. J., S4.117

Criollo-Chan, M. A., S1.2

Cristóbal-Alejo, J., S1.2

Cristofani-Yaly, M., 518

Croce, V., S4.31

Crosby, K., S4.27

Crosby, K. M., S4.5

Crouch, J. A., S3.2, S4.88, S4.119, S4.122, S4.124, S4.168

Crowhurst, R. N., 863

Crowley, M., S4.92

Crutcher, F. K., S4.31

Cruz, C., S4.32

Cruz, D. R., S4.31

Cruz, L., S2.2, S4.121

Csinos, A. S., S2.9

Cubeta, M., S4.100

Cubeta, M. A., 533, S4.126
Cucmis melo: Cucurbit aphid-borne yellows virus resistance in accession TGR-1551, 1389

Cucurbit aphid-borne yellows virus: resistance in melon accession TGR-1551, 1389

Cucurbit downy mildew. See Pseudoperonospora cubensis

Cuevas, O., S4.78

Cui, B., S4.32

Culbreath, A., S2.1, S2.12, S4.130

Culbreath, A. K., S2.6, S2.9, S2.11

Culman, S., S4.41

Cummings, J. A., 695

Cummings, T., S4.46

Cunnac, S., S4.37, S4.136

Curry, S., S4.19

Cursino, L., S4.57

Cynodon spp.: infection and colonization by

Ophiosphaerella korrae, 656

CYP51 gene, 370

Dader, B., S4.32

Dagno, K., S4.136, S4.149

Da Graça, J. V., S4.27, S4.32

Dai, R., S4.75

Dailey O’Brien, D., S4.130

Dalisay, T. U., S4.67

Dalla Lana, F., 307, S4.33

Dallot, S., 1408

Dally, E. L., S4.33

Dalsing, B., S4.72, S4.139

Dalsing, B. L., S4.85

Damacena, M. B., 1229

Damann, K. E., S4.33, S4.134

Damann, K. E., Jr., S2.10

DaMatta, F. M., 180, 738

Damicone, J., S2.3, S4.25

Dang, T., S4.33, S4.50, S4.135

Dangl, J., S4.173

Daniel, R., S4.179

Daniels, J., S4.124

Danies, G., 342, 966, S4.25, S4.33

Dao, S., S4.136

Das, J., S4.167

Da Silva, M. P., S4.32

Da Silva, W. L., S4.32

Dassanayake, M., S2.5, S4.127

Daubert, S., 758, S4.5

Daudi, H., S4.63

Daughtrey, M., S4.130

Daughtrey, M. L., 388, S1.6

Daunay, M.-C., 597, 1529

Davenport, B., S4.158

David, D. R., 1059

Davis, C., S4.162

Davis, D., S4.58

Davis, E. L., 1362

Davis, R., S4.13

Davis, R. E., S1.1, S1.2, S4.33

Davis, R. F., 1522

Davis, R. M., S4.31, S4.37, S4.139

Dawson, J., S4.179

de Almeida-Engler, J., 628

Dean, R. A., S4.176

Deblasio, S. L., S4.34

Debona, D., 1050, S4.34

Debonneville, C., S4.34

Dee, M. M., S4.30, S4.127

Defense-related gene expression: light-induced upregulation in tomato infected with

Pseudomonas cichorii JBC1, 412

DeGaetano, A., S4.24

Deisher, D., S4.34

De Jesus-Kim, L., S4.156

De Jonge, R., S4.15, S4.17

De Koeyer, D., S4.101

De La Fuente, L., 855, S2.2

Delaunay, A., 1408

Del Castillo Munera, J., S4.34

Delfosse, P., S4.28, S4.40 
Delgado, J. A., S4.34

Delisle-Houde, M., S4.35

Delledonne, M., 199

del Mar Jimenez-Gasco, M., S4.109

Delong, J., S4.35

Delorean, E. E., S4.18

Del Ponte, E. M., 246, 307, 441, 695

DeLury, N., S4.112

Dembele, M., S4.136

Demers, J., S4.35, S4.88, S4.123

Deng, W. L., S4.29, S4.35, S4.133, S4.151

Deng, X., S4.157, S4.159

Dennis, G., S4.130

de Oliveira, S. C., 284

Deora, A., S4.52, S4.92

Deoxynivalenol: effects of post-anthesis moisture patterns on accumulation in wheat, 210

Derevnina, L., S4.169

DeRobertis, C., S4.33

Desaeger, J. A., S3.1, S3.3

Deschamps, S., 1362

Desitter, T., S4.20

de Souza, J., S4.143

Dessaux, Y., S4.118

de Vallavieille-Pope, C., S4.142

Devanaboina, M., S4.142

DeVetter, L. W., S4.147

Dewdney, M. M., S2.3, S4.71, S4.101, S4.145, S4.157

De Wolf, E. D., S4.34, S4.44, S4.52

DeYoung, R., S4.151

Dhillon, B. D., S4.172

Diaporthe spp.: reemergence as a threat to sunflower, 990

Diarra, B., S4.138

Diaz, C., S4.25

Diaz, C. I., S2.3

Diaz, G., S4.84

Diaz, G. A., S4.36

Diaz, J. E., S4.71

Diaz-Lara, A., S4.36

Di Bello, P. L., S4.36

Dichorhavirus, 564

Dickison, V., S4.101

Diedhiou, P. M., S4.63

Diffusible signal factor (DSF)-controlled virulence genes: in Xanthomonas oryzae pv. oryzidola Rs105, 588

Dillard, H. R., S4.51, S4.76

Dinesh-Kumar, S. P., S4.105, S4.174

Ding, F., S3.2, S4.36

Ding, S., S4.36, S4.37

Ding, S. W., S4.161

Dinglasan, J., S4.62

Disease control and pest management:

characterization of $\mathrm{Cr} 811$ gene in pathotype 5 of Plasmodiophora brassicae, 764; discoveries and challenges in control of basil downy mildew, 885; effect of maize hybrid and foliar fungicides on yield, 1080; effect of Raffaelea lauricola on avocado leaf gas exchange and xylem sap flow, 433; effect of shoot potassium concentration on gray mold in sweet basil, 1059; effects of maple bark biochar on Rhizoctonia solani metabolism and damping-off severity, 1334; emergence and spread of new races of wheat stem rust fungus, 872 ; emergence of fungicide resistance in Botrytis cinerea, 424; information graphs for binary predictors, 9; inhibition of Phytophthora parasitica and $P$. capsici by silver nanoparticles, 1183; knock-down of HSP90 and isocitrate lyase gene expression reduces root-knot nematode reproduction, 628; next-generation sequencing versus biological indexing for detection of grapevine viral pathogens, 758; physiological responses of hard red winter wheat to Wheat streak mosaic virus infection, 621; predicting cereal root disease using soil DNA and environmental parameters, 1069; QoI resistance in Brazilian populations of Magnaporthe oryzae,
284; review of Ramularia collo-cygni, 895; STEMRUST_G model, evaluation and implementation, 35

Ditta, S., S4.165

Dittrich, J., S4.36

Ditylenchus destructor: resistance in sweet potato by

the expression of siRNAs targeting unc-15, 1458

Dixon, E., S2.7, S4.146

Dm50 gene, 1220

DNA macroarrays: detection and identification of fungal pathogens causing decline of young grapevines, 1373

Doan, H., S4.31

Doan, H. K., 1036, S4.37

Dobinson, K. F., S4.176

Doll, D., S4.13, S4.37

Domiciano, G. P., 738

Domier, L. L., S4.27, S4.90

Domingo, S. J., S4.55

Dominguez-Serrano, D., S4.48

Donda, B., S4.37

Dong, H.-T., 220

Dong, Y., 441, S4.16

Donofrio, N. M., S3.1, S3.4, S4.33, S4.95, S4.122, S4.180

Donovan, D., S4.75

Dorigan, A. F., 284

Dornelas, G. A., S4.27

Dorrance, A. E., S4.41, S4.49, S4.81, S4.85

Dossa, G. S. C., S4.37

Dossman, B. C., S4.85

Dothistroma septosporum: sporal dispersal in northwest British Columbia, 69

Dou, F., S4.159

Doucoure, H., S4.37, S4.136

Dougoud, J., S4.89

Douhan, G., S4.92

Douhan, G. W., S4.141

Doumbia, M., S4.136

Doussoulin, H. A., S4.98

Dracatos, P., 490

Dragich, M. A., S4.38

Dreaden, T., S4.38, S4.129

Drenth, A., S4.4, S4.166

Drew, A., S2.8

Druebbisch, B., S4.4, S4.56, S4.76, S4.135, S4.148

Dry, I. B., 1104

D'Souza, D. H., S4.20

Du, L., 1318

Du, Z., S4.3

Duan, S., S4.38

Duan, Y., S4.36, S4.38, S4.76

Duan, Y. P., S4.71

Dubcovsky, J., 1347

Dufault, N. S., 230, 905, S2.1, S2.8, S4.141

Dugan, F. M., S4.38

Dugyala, S., 707, S4.160

Duna, Y., S4.153

Dundore-Arias, J. P., S4.39

Dung, J., S4.70

Dung, J. K. S., S4.124

Dunn, A. R., 1355, S4.51

Durvasula, A., S4.5

Dusunceli, F., S4.166

Dutheil, J. Y., S4.172

Dutta, B., S2.3, S2.9, S4.39

Dutta, M., S4.39

Dyer, A., S4.54

Easter, M., S4.164

Eastwell, K. C., 399

Ebadzad, G., S4.39

Eckshtain-Levi, N., S4.40

Ecology and epidemiology: assessing the mismatch between incubation and latent periods of vector-borne diseases, 1408; Dothistroma septosporum sporal dispersal, 69; effect of Brassica seed meal soil amendments on apple production, 460; effect of temperature on growth and sporulation of Phytophthora infestans US-22, US-23, and US-24 clonal lineages, 449; effects of Fusarium head blight on grain yield and test weight in soft red winter wheat, 295; effects of host genotype and hypersensitive reaction on Xanthomonas campestris pv. vitians in lettuce, 316; effects of post-anthesis moisture patterns on Fusarium head blight development and DON accumulation in wheat, 210; effects of wheat residue on severity of Stagonospora nodorum blotch and winter wheat yield, 1417; emerging threat of maize lethal necrosis in Sub-Saharan Africa, 956; environmental and pathogenic factors inducing brown apical necrosis on English walnut fruit, 1427; environmental conditions affect Botrytis cinerea infection of grape berries more than strain or transposon genotype, 1090; evaluation of BlightPro decision support system for management of potato late blight, 1545; factors affecting Botrytis cinerea infection on grapevine inflorescences and young berry clusters, 325; factors affecting dynamics of Verticillium microsclerotia in field soils, 658; Fusarium graminearum cross-species pathogenicity testing, 441 ; interactions between cassava brown streak disease and Bemisia tabaci, 646; meta-analysis of soybean rust severity and crop yield, 307; phenotypic and genotypic characterization of Puccinia graminis f. sp. tritici race TKTTF, 917; Phytophthora alni subspecies distribution in southern Sweden, 1191; population structure of Phytophthora infestans in a potato germplasm nursery, 771; potential effects of diurnal temperature oscillations on potato late blight, 230; response to Phakopsora pachyrhizi in North America, 905; significance of late-season infections in management of potato zebra chip, 929; simulation model for stem rust epidemics in ryegrass seed crops, 45; soybean rust: history, epidemic evolution, and model burn-in for a network of annual invasion, 947; systemic infection of Puccinia horiana in chrysanthemum, 91, cover photo: January; temporal epidemiology of sudden oak death in Oregon, 937;

Verticillium in spinach fields and transmission to subsequent lettuce crops, 80; waterborne Phytophthora ramorum, detection, diversity, and population dynamics, 57; wet-sieving qPCR detection of Verticillium dahliae, 220

Edae, E., S4.68

Edison, G., S4.124

Edlebeck, K., S4.104

Edwards, J., S4.52

Edwards, J. T., S2.4

Edwards, M. C., 833, S4.85, S4.86

Edwards, P., S4.19

Edwards, S., 1174

Edwards, T. P., S4.10

Ehlert, K., S4.53

Ehteshamul-Haque, S., S4.10, S4.40, S4.102

Eisenback, J., S4.142

Elad, Y., 1059

Elafifi, S., S4.40

Elena, S. F., 18

El Jarroudi, M., S4.28, S4.40

Elkahky, M. T., S4.40

Elliott, S., S4.42

Ellis, M. L., S4.40

Ellison, M. A., S3.3

Elmarakeby, H. A., S4.143

Elmazaty, M., S4.40

Elmer, W., S1.7

Elphinstone, J., S4.5

Elsheshtawi, M., S4.40

Emerson, M., S2.5

Emmanuel, C. J., S4.41

Emmitt, R. S., S2.3 
Ernest, E. G., S3.1, S3.4, S4.95, S4.180

Erwinia amylovora: characterization of streptomycin resistance in California isolates, 1302

Erysiphe necator: mechanisms of azole resistance, 370; quantitative resistance to by Vitis rupestris B38, 1097; strategies for RUN1 gene deployment to manage in grapevine, 1104

Eskalen, A., S4.91, S4.92, S4.180

Esker, P. D., 1080, 1400, S4.126

Eskridge, K. M., 786, S4.47

Espíndola, A. S., 684, S4.4

Estévez de Jensen, C., S1.2

Etiology: characterization of Alternaria spp. associated with fruit rot of blueberry in California, 1555; infection and colonization of bermudagrasses by Ophiosphaerella korrae, 656; reasons to consider Phytophthora infestans a reemerging pathogen, 966

Eujayl, I. A., S4.132

Eutypa lata: molecular polymorphism and phenotypic diversity, 255

Evans, P., S4.87

Evans, T. A., S3.4, S4.95, S4.180

Everhart, S., S4.7

Everhart, S. E., 542, S4.41

Eversole, K., S4.106

Everts, K. L., 966, S3.4, S4.180

Eyre, C. A., 57

Eyre, M., S4.41

Fábián, A., 797

F-actin dynamics: in Phytophthora infestans, effect of flumoroph on, 419

Faingold, I., 1059

Faino, L., S4.17

Fairchild, K., S4.37, S4.148, S4.149

Fajardo, J., S4.106

Fakhoury, A., S4.90, S4.131, S4.146, S4.156

Fakhoury, A. M., 1601, S4.63, S4.134

Falach, L., 778

Falconi, C. E., S4.153

Falk, B., S4.103, S4.111, S4.161, S4.170

Falkenberg, P., S4.41

Falkenstein, K., S3.5

Fan, R., 220

Fan, W., 1458

Fan, Z., S4.42

Fang, A., S4.175

Faris, J. D., S4.69, S4.74

Farman, M. L., S4.111

Farrar, J., S4.42

Farzaneh, M., S4.90

Faske, T. R., S2.5, S4.181

Faura, A., S4.123

Faure, C., 135, 1155

Faure, D., S4.118

Fazio, G., S4.159

Feau, N., S4.172

Fedynich, A., S4.124

Feechan, A., 1104

Fefer, M., S4.92

Fei, Z., 342, S4.70, S4.106

Feliciano, M., S1.4

Feliciano-Rivera, M., S1.2, S1.3

Fellers, J., S4.70

Fellers, J. P., S4.121

Feng, C., S4.42, S4.109, S4.120

Feng, J., 764, S4.42

Feng, J. Y., 1206

Feng, X., 1487, S4.42

Fennessey, C. M., S4.43, S4.73

Fennimore, S., S4.37

Fenoglio, J. J., S4.109

Fereres, A., 1389, S4.32

Fermaud, M., 325

Fermaud, N., 1090

Fernandes, S. V., S4.131

Fernandez, E., S4.43
Fernández-Ortuño, D., 424, S4.61

Fernando, D., S4.43

Ferrandino, F. J., S1.7

Ferrão, M. A. G., 805

Ferrari, A., 199

Ferrarini, A., 199

Ferreira, M. A., 1229

Fetch, T., 728

Fetch, T., Jr., 490

Fiafia, S. T., S4.9

Fichtner, E., S4.52

Fichtner, E. J., S4.43

Fiers, M. W. E. J., 863

Filiatrault, M. J., S4.44

Filion, M., 1311

Filippi, M. C. C., 738

Filloux, D., S4.43

Fiorin, A., 1427

Fischer, G., S4.163

Fischer, T., S4.44

Fisher, A., S4.136

Fishman, M., S4.44

Fitzgerald, G., S4.139

Fleites, L. A., S4.44, S4.64

Fletcher, J., S4.97

Florence, J., S4.44

Flores, F. J., 656

Flores-Gonzalez, E., S1.2

Floyd, C. M., 1601, S4.44

Flumorph: effect on F-actin dynamics in

Phytophthora infestans, 419

Fluorescence microscopy: analysis of wheat stem

infection process on barley, 707

Fobert, P., S4.107

Fobert, P. R., S4.77

Folimonova, S. Y., S4.15

Fondong, V., S4.174

Fonseca, N. R., S4.44

Font i Forcada, C., 1220

Forbes, G., S4.137

Forbes, G. A., S4.59

Forcelini, B. B., S4.45

Ford, B., S3.1

Ford, R., 358

Forster, H., S4.3, S4.52, S4.55, S4.101, S4.168

Förster, H., 1302, S4.57

Fortunato, A. A., 1050

Foster-Frey, J., S4.75

Fountain, J. C., S2.3, S2.13, S4.154, S4.175

Fourie, P. H., S4.71

Fox, A., S4.176

Fraedrich, S. W., S4.15, S4.45, S4.151

Fragoso, R. R., 628

Fraisse, C., S4.171

Frampton, R. A., 863

France, R. A., S4.45, S4.53

Francis, D. M., 126, 1437

Franco, B., S4.45

Franco, J. G., S4.51

Franco, M. E., S4.45, S4.46

Frankel, S. J., S4.164, S4.165, S4.170

Frantz, G., 388, S4.11

Frederick, Z., S4.46

Freeman, A., S4.139

Freeman, J. H., S2.8

Frei, P., 895

Freitas, C. C., 738

French-Monar, R. D., S1.4

Frenkel, O., 370

Frey, J. E., 1245, 1262

Frey, T. S., S4.46

Frey, W. E., 342, S1.7

Frias, A. A., S4.51

Friesen, T., 500, 509

Friesen, T. L., S4.47, S4.74

Frost, K., S4.70

Frost, K. E., 449, S4.124

Fry, W., S4.104

Fry, W. E., 966, 1545, 1594, S4.33, S4.129
Fu, S., 1277, S4.57

Fuchs, M., S4.137

Fuchs, M. F., 1026

Fuentes Aponte, S. E., S1.2

Fuentes-Bueno, I., S4.47, S4.55

Fujita, S., S4.46

Fulbright, D. W., S4.93

Fulcher, M., S3.3

Fulladolsa, A. C., S4.46

Fultz, F. E., S2.4

Fultz, L., S4.16

Fumonisin: assessment of contamination of maize

in western Kenya, 1250

Funahashi, F., S4.46

Funderburk, J. E., 388, S4.11

Fungicide resistance: emergence in Botrytis cinerea, 424

Funnell-Harris, D. L., 786, S4.47

Furlanetto, C., 805

Fusarium head blight (FHB): effects of post-anthesis moisture patterns on development and DON accumulation in wheat, 210; effects on grain yield and test weight in soft red winter wheat, 295; factors affecting the composition of pathogens in a subtropical no-till wheat agroecosystem, 246; genetic architecture of resistance in triticale, 334. See also Fusarium graminearum

Fusarium spp.: environmental and pathogenic factors inducing brown apical necrosis on English walnut fruit, 1427; mutation in MILDEW LOCUS $O$ of barley does not affect resistance to grain infection, 1214

-F. asiaticum, 246

-F. austroamericanum, 246

-F. circinatum: effects of resin and monoterpenes on spore germination and growth, 119

-F. cortaderiae, 246

-F. culmorum: genetic architecture of resistance in triticale, 334 (see also Fusarium head blight)

-F. graminearum: cross-species pathogenicity testing, 441; population subdivision from barley and wheat in the upper midwest U.S., 1466; species complex, factors affecting the composition of pathogens in a subtropical no-till wheat agroecosystem, 246; trichothecene genotype composition of populations in New York, 695 (see also Fusarium head blight) -F. meridionale, 246

-F. oxysporum f. sp. cepae: genetic and pathogenic variability in onion and Welsh onion, 525

-F. oxysporum f. sp. cubense: Fusarium wilt of banana, review article, cover photo: December, 1512

-F. pseudograminearum: predicting disease with soil DNA and environmental parameters, 1069

-F. temperatum, 119

-F. virguliforme: improved diagnosis and quantification of, 378; multilaboratory comparison of qPCR assays for detection and quantification, 1601

Fusarium wilt of banana. See Fusarium oxysporum f. sp. cubense

Gabriel, D. W., S4.44, S4.64

Gadagkar, S., S4.137

Gadoury, D. M., 1104, S4.9, S4.178

Gaeumannomyces graminis var. tritici: predicting disease with soil DNA and environmental parameters, 1069

Gager, J., S1.7, S4.77

Galarneau, E., 1097, S4.47, S4.78

Gale, S., 917

Galeano, C. H., 1220

Gallant, S. L., S4.71

Gallitelli, D., 1155

Galván, M. Z., S4.50

Gambone, K., S4.47

Gamliel, A., S4.97 
Gañan, L., S1.1, S4.47, S4.180

Gandra, P., S4.10

Gang, C. H., S4.144

Gao, L., 1131

Gao, M., S4.48

Gao, S., S4.47, S4.48, S4.158

Gao, W., S4.51

Gao, Y., S4.47

Garampalli, R. H., S4.48

Garavito, M., S4.113

Garbelotto, M., 57

Garbelotto, M. M., S4.27

Garcia, C., S4.14, S4.40

Garcia, T. G., S4.128

Garcia Aroca, T. G., S2.2, S2.11

García-Velasco, R., S4.48

Garfinkel, A. R., S4.48, S4.49

Garibaldi, A., S4.53, S4.114

Garrett, C., S2.7

Garrett, K., S4.113, S4.137, S4.179

Garrett, K. A., 947, S4.59

Garrett, W., S4.43

Garrido, P., 684

Garvin, D. F., 482

Garzo, E., 1389

Garzón, C. D., 684, S4.41, S4.66, S4.97, S4.105, S4.109

Gaskins, V. L., S4.67, S4.87

Gasoni, L., S4.123

Gasparich, G. E., S4.33

Gauthier, N. W., S2.7

Gaynor, B. J., S4.33

Geary, B., S4.49, S4.58, S4.134

Geary, B. D., S4.16, S4.64

Gebben, S. J., 316

Gebreil, A., S4.90, S4.103

Gedling, C. R., S4.49

Gee, C. T., S3.3

Geisler, M., S4.134

Gene expression profiling: of Ampelomyces quisqualis during host-induced germination, 199

Genetics and resistance: association analysis for bacterial spot resistance in tomato, 1437; basil downy mildew resistance in Ocimum species, 778; Brachypodium distachyon-Cochliobolus sativus pathosystem, 482, cover photo: April; downy mildew resistance in lettuce ' $\mathrm{La}$ Brillante' conferred by Dm50 gene and multiple QTL, 1220; effect of waxy gene on fungal infection in sorghum grain, 786; genetic architecture of Fusarium head blight resistance in triticale, 334; grapevine powdery mildew, strategies for RUN1 gene deployment to manage, 1104; identification of a QTL for plant height in sweet sorghum, 1522; incidence of 'Candidatus Liberibacter asiaticus' in citrandarins, 518; inheritance and identification of a major QTL for Meloidogyne incognita resistance in sweet sorghum, 1522; MILDEW LOCUS $O$ mutation does not affect resistance to grain infections with Fusarium spp. and Ramularia collo-cygni, 1214; Phytophthora fruit rot resistance, worldwide evaluation of Capsicum annuum for, 110; Phytophthora infestans, acquired mefenoxam resistance in, 342; Phytophthora infestans resistance, testing taxonomic predictivity in wild Solanum spp., 1198; potato tuber blight resistance, age-dependent correlation with $R B$ transgene transcript levels, 1131; powdery mildew race $1 \mathrm{~W}$ resistance in watermelon, inheritance of, 1446, cover photo: November; Puccinia graminis f. sp. avenae resistance gene Rpg5 in barley, 490; Pyricularia oryzae resistance gene Rmg7 in tetraploid wheat, 495; Pyricularia oryzae resistance gene Rmg8 in hexaploid wheat, 1568; QTL meta-analysis of leaf rust resistance in wheat, 1585; QTLs from oat genotypes conditioning resistance to Puccinia coronata, 239; quantitative resistance to Erysiphe necator by Vitis rupestris B38, 1097; rpg4/Rpg5 locus in resistance to wheat stem rust race TTKSK in wild barley, 99; specificity of the 7DL rust resistance suppressor in Canthatch spring wheat, 477; spot form net blotch resistance, evaluation of a barley core collection for, 509; spot form net blotch seedling resistance in barley, worldwide association mapping, 500; stem rust resistance gene SrND643 in wheat, mapping and validation of, 470 ; stem rust resistance gene $\operatorname{SrTm} 4$, mapping of, 1347; stripe rust resistance, characterization in wheat lines with resistance gene $\operatorname{Yr} 17,1123$; stripe rust resistance gene $\operatorname{YrSP}$, mapping in wheat, 1206; stripe rust resistance in wheat, race-specific, 1114

Gene transfer: with Agrobacterium tumefaciens, review article, 1288

Genger, R. K., S4.179

Genin, S., S4.118

Gent, D., S4.24, S4.49, S4.114

Gerard, P., 674

Gerberich, K., S4.150

Gershovitz, M., S4.40, S4.49

Geske, A. P., S4.89

Gessler, C., 199

Gevens, A., S4.31, S4.37

Gevens, A. J., 449, 966, S4.36, S4.89, S4.146

Ghimire, S., S4.49

Ghosh, D. K., 1043

Ghosh, D. K., Sr., 1043

Giampetruzzi, A., 555

Gibberella ear rot. See Fusarium graminearum

Gibson, R. W., S4.179

Giesbrecht, M., S4.105

Giesler, L., S4.173

Giesler, L. J., 905, S4.99

Gigot, C., S4.50, S4.142

Gil, J., S4.169

Gilardi, G., S4.53

Gilbert, M. T. P., S4.118

Gilbertson, R. L., 141, S4.13, S4.28, S4.87, S4.90, S4.104, S4.143

Giles, K., S4.167

Gill, U., S4.50

Gill, U. S., 580

Gilley, M., S4.50

Gilligan, C. A., 917

Gingrich, G. A., 35

Ginnan, N. A., S4.50

Gisi, U., 998

Gitaitis, R., S2.3, S4.39

Gitaitis, R. D., S2.9

Glassy-winged sharpshooters. See Homalodisca vitripennis

Glawe, D. A., S4.60

Gleason, M. L., S4.59

Glenn, A. E., S4.17, S4.48

Glenn, D. M., S4.64

Glenn, T., S4.132

Global climate change: potential effects of diurnal temperature oscillations on potato late blight, 230

Glover, K., S4.2

Gludovacz, T., S4.92

Glycine max: antioxidant system changes in leaves infected by Corynespora cassiicola, 1050; diagnosis of Rhizoctonia solani and Macrophomina phaseolina in using LAMP assays, 1612; multilaboratory comparison of qPCR assays for detection and quantification of Fusarium virguliforme, 1601

Godfrey, K., S4.97

Godoy, C. V., 307

Godoy-Lutz, G., S4.131

Goenaga, R., S1.4

Gold, S. E., S4.16, S4.48, S4.117

Golden, B., S2.12, S4.148
Golden, B. R., S4.68, S4.88

Goldman, P., S4.5

Goldman, P. H., 316

Golino, D., 758, S4.5, S4.8

Gomes, A. C. M. M., 805

Gomes, J. G., S4.34

Gomes, L. B., 246

Gómez-Guillamón, M. L., 1389

Gómez-Mercado, R., S4.141

Gonçalves-Vidigal, M. C., S4.50

Goncalves-Zuliani, A. M., S4.51

Gong, J. S., S4.83, S4.108

Gong, L., S2.4

Gong, R., S4.62

Gongora-Castillo, E., S4.114

Gonzalez, A., 840, S4.11

Gonzalez, L. N., S4.25

González, M., S4.31

Gonzalez Anta, G., S4.123

Gonzalez-Diaz, J. G., S4.48

Gonzalez-Garcia, L. N., S4.33

Gonzalez Velez, A., S1.2

Goodin, M. M., S4.89

Goodwin, P., S4.51

Goodwin, S. B., S4.176

Gordon, A., S4.95

Gordon, T. C., 239

Gordon, T. R., 119, S4.58, S4.108, S4.125

Gorny, A. M., S4.51

Gorshkov, V., S4.97

Gosalvez, B., 1389

Goss, E. M., 230, S2.8, S4.74, S4.101, S4.141, S4.145, S4.157

Gossen, B. D., S4.26, S4.51, S4.52, S4.92

Gossypium hirsutum: factors affecting the spatial

distribution of reniform nematode in, 674

Gottwald, T., S4.7, S4.86, S4.87, S4.93

Goulding, C., S4.162

Gouran, H., S4.52

Govers, F., 419

Gowda, P., S4.167

Gowda, S., 1043

Goyer, C., 1311

Grabke, A., 424

Grabow, B. S., S4.52

Grady, K., S4.42

Graf Grachet, N., S2.4, S4.52

Graham, J. H., S4.66, S4.99, S4.150

Grandaubert, J., S4.172, S4.176

Grant, J., S4.126

Grapevine leafroll-associated virus 4: biological,

serological, and molecular characterization of, 1262

Grapevine leafroll disease, 1262

Grapevine Pinot gris virus: genetic variability and association with grapevine leaf mottling and deformation, 555

Grapevine powdery mildew. See Erysiphe necator

Grapevine red blotch-associated virus: emerging

threat of, 1026, cover photo: July

Grau, C. R., S4.156

Graves, E., S4.146

Gray, M. A., S4.52

Gray, S., S4.115

Gray, S. M., S4.32, S4.34, S4.152

Graybosch, R. A., 1496, S4.54

Gray mold. See Botrytis cinerea

Greenhut, I. V., S4.53

Greenspan, A., S4.5

Greer, G., S4.135

Gregg, L., S4.5

Gregory, N., S4.126

Gregory, N. F., S3.3, S4.95, S4.180

Grenell, A., S4.144

Grigoriev, I., S4.171

Grijalva, A. C., S4.153

Grimberg, B., S4.53

Grinbergs, D. E., S4.45, S4.53

Grosser, J., S4.99

Grosser, J. W., S4.56, S4.66 
Grossi-de-Sa, M. F., 628

Groth, D., S2.9

Groundnut ringspot virus: emergence in southeastern U.S., 388, cover photo: March

Grove, G. G., S4.60

Groves, R., S4.179

Groves, R. L., 825, S4.16, S4.39

Grünwald, N. J., 684, 966, 982, S4.68, S4.76, S4.110, S4.134, S4.172

$\mathrm{Gu}, \mathrm{X} ., \mathrm{S} 4.42$

Gualandri, V., 555

Guaragna, M. A., S4.67

Gudmestad, N. C., 863

Guenther, R. H., S4.53

Guerrero-Turriza, H. O., S1.2

Guest, D. I., S4.179

Gugino, B. K., 966, S4.109

Guidot, A., 1529

Guimarães, E. A., S4.130

Guimaraes, L. M., S4.44

Guimarães, R. J., S4.27

Gullino, M. L., S4.53, S4.54, S4.114

Gulya, T. J., 990

Gummy stem blight: genetic diversity and population structure, 815

Gumus, M., S4.22

Gunnink Troth, E., S4.54

Guo, B., S2.3, S2.11, S2.13, S4.154, S4.175

Guo, H., S4.54

Guo, L., 885, S4.54

Guo, Q., S4.75

Guo, X., 1458

Guo, Y. Y., S4.164

Gupta, A., S4.54

Gurr, B., S4.9

Gurung, S., 80, 638, S4.3

Gutierrez, O., S4.20

Gutierrez, O. A., S4.111

Guzmán, M. L., S4.23

Gwinn, K. D., S4.20, S4.30, S4.55, S4.75

Haack, S., S4.55

Haag, P., 1373, S4.140

Haak, D. C., S4.93

Hadad, R., S1.5

Hadziabdic, D., S4.55

Hagan, A., S2.4

Hagan, A. K., S4.55

Hahn, J. P., S4.55

Haider, M. S., S4.159

Hailu, E., 917, S4.65

Hajeri, S., S4.154

Haji, S., 646

Halbert, S., S4.100

Halbert, S. E., S4.71, S4.115

Hall, D. G., S4.115

Hall, M. E., S4.56

Hallin, E., S4.77

Halterman, D., S4.3, S4.28, S4.83, S4.175

Halterman, D. A., 1198, S4.46

Ham, J. H., S4.109, S4.127

Hambleton, S., S4.85

Hameed, U., S4.159

Hamelin, R. C., S4.172

Hamill, J. E., S4.56

Hamm, P., S4.70

Hamm, P. B., S4.124

Hammill, D. L., S4.152

Hammond, J., S4.11, S4.56, S4.67, S4.83, S4.108, S4.125

Hammond, R. W., S4.75, S4.174

Hammons, B. W., S4.31

Hamza, S., S4.8

Han, C. U., S4.69

Han, J., S4.56

Han, S., S4.65

Han, Y., S4.175

Han, Z., S4.164

Handique, U., S4.56, S4.118
Hanna, J. W., S4.16

Hans, G. R., S4.9

Hansen, E. M., 937, 982, S4.68

Hansen, M. A., S3.3

Hansen, Z. R., S4.177

Hanson, B., S4.37

Hanson, L. E., S4.56

Hao, B., 621

Hao, J., S4.65, S4.94

Hao, L., S4.57

Hao, W., S4.52, S4.57

Harding, M. W., S4.26

Harmon, P. F., S4.131

Harries, M., 1069

Harrington, T. C., 1229, S4.45, S4.151, S4.169

Harrison, A., S4.57, S4.137

Harrison, N. A., S4.33

Harrison, S. A., 239

Harris-Schultz, K. R., 1522

Hartman, G. L., 905, 1601, S4.27, S4.90, S4.109

Hartung, J., S3.2, S4.36

Hartung, J. S., 564, 1013, 1277, S1.3, S4.57, S4.121

Harveson, R., S4.50

Harveson, R. M., S4.58, S4.146

Harvey, J., S4.120

Harvey, J. W., 1250

Harvey, R., S4.58

Hasey, J., S4.52

Hashiguti, H. T., S4.51

Hashimoto, K., S4.70

Hasiów-Jaroszewska, B., 1270

Haskell, J., S4.56, S4.135

Hassell, R., S4.75

Hatch, P., S4.49, S4.58

Haudenshield, J. S., 1601

Hausbeck, M., 998, S4.169

Hausbeck, M. K., 110, S4.34, S4.99, S4.109, S4.149

Havis, N. D., 895

Hawara, E., S4.107

Hawes, M., S4.138

Hawkins, G., S4.101

Hay, F., S4.125

Hay, F. S., 358

Hayashi, Y., S4.58

Hayes, B., 597

Hayes, C., S4.162

Hayes, R. J., 316, 638, 662, 1220, S4.116

Haygood, R., S2.4

Hayslett, M., S4.58

$\mathrm{He}, \mathrm{C} ., \mathrm{S} 4.82$

Heath, L. S., S4.143

Heat shock proteins: knock-down of HSP90 gene in root-knot nematode reduces reproduction, 628; overexpression in Agrobacterium tumefaciens promotes virulence in Arabidopsis, 160

Heat-stable antifungal factor: PilG regulation in Lysobacter enzymogenes, 1318

Hegarty, J. F., S4.126

Hein, G. L., 1496, S4.54, S4.150

Helianthus annuus: reemergence of Phomopsis stem canker, 990

Hemileia vastatrix, cover photo: September; review article, 1164

Hendricks, K. E., S2.5

Henke, S., S4.124

Henry, A., S4.37

Henry, P., S4.58

Hernandez, E., S4.59

Hernandez-Cruz, A, S4.59

Hernandez-Matinez, R., S4.107

Hernandez Nopsa, J. F., S4.59

Herrera, F. A., S4.23

Herrera-Estrella, A. E., S4.107

Herrera Paredes, S., S4.173

Herrmann, H. W., S4.20, S4.159

Hess, M., 895, 1214
Heterodera glycines: new candidate effectors produced in esophageal gland cells, 1362, cover photo: October

Hetherington, F., S4.149

Hewavitharana, S. S., 460, S4.59

Hewezi, T., 1362, S4.111

Hibiscus green spot virus 2, 1013

Higgins, R., S4.41

Hill, C. B., S4.109

Hill, M. A., S3.3, S4.126

Hillis, V., S4.13

Hincapie, M., S4.60

Hinton, D. M., S4.10

Hirayama, Y., S4.9

Hirnyck, R., S4.42

Hisano, S., S4.157

Hladky, L. L., S4.13

Ho, T., S2.5, S4.60

Hochmuth, B., S2.8

Hockemeyer, K., S4.60

Hodson, D., 917, S4.65

Hodson, D. P., 872

Hofer, K., 1214

Hoffmann, V., 1250

Hofseth, L., S4.162

Holbrook, C., S2.11

Holguin, C. M., 674

Holladay, W. T., S4.102

Holland, L. A., S4.60

Holland, R., S4.19

Holland, R. T., S4.156

Hollier, C., S2.9, S4.86, S4.112

Holman, D., S4.167

Holmes, G., S4.25

Holshouser, D., S4.94

Homa, K., 885

Homalodisca vitripennis: direct evidence for egestion and salivation of Xylella fastidiosa, 608 Honesty, S., S4.75

Hong, C., S4.10, S4.30, S4.154

Hong, J., S4.26

Hong, J. C., S4.60, S4.120

Hong, L. L., S4.83

Hopkins, B. G., S4.16

Hordeum vulgare: MILDEW LOCUS O mutation does not affect resistance to grain infections with Fusarium spp. and Ramularia collo-cygni, 1214; population subdivision of Fusarium graminearum in the upper midwest U.S., 1466; Puccinia graminis f. sp. avenae resistance gene Rpg5, 490; qPCR and fluorescence microscopic analysis of wheat stem rust infection process, 707; review of Ramularia collo-cygni, 895; spot form net blotch resistance, evaluation of a core collection for, 509; spot form net blotch seedling resistance , worldwide association mapping, 500

-H. vulgare subsp. spontaneum: resistance to wheat stem rust race TTKSK, 99

-H. vulgare subsp. vulgare: resistance to wheat stem rust race TTKSK, 99

Hort, M., 917

Host resistance: versus nonhost resistance, review article, 580

Hotchkiss, M. W., S4.17, S4.27

Hovmøller, M. S., 872, 917

Howe, K., S4.97

Hoy, J., S4.16

Hoyt, P., S4.41

Hoyt, P. R., S2.12

hshA gene, 588

$h s h C$ gene, 588

Hsieh, W. H., S4.77

Hsu, J. C., S4.83

$\mathrm{Hu}$, C. H., S4.84

$\mathrm{Hu}$, D., S2.5, S4.61

Hu, J., S4.61, S4.80, S4.135, S4.157

$\mathrm{Hu}$, M., S4.63

Hu, M. J., S4.61, S4.124

$\mathrm{Hu}, \mathrm{T} ., \mathrm{S} 4.175$ 
$\mathrm{Hu}, \mathrm{X}, 1174,1325$

Hu, X. P., S4.61

$\mathrm{Hu}$, X.-P., 220

Hua, C., 419

Huang, C., S4.175

Huang, F.-C., 160

Huang, J. W., S4.26, S4.35, S4.151

Huang, L., 477

Huang, Q., S4.132

Huang, S.-C., 160

Huang, T. C., S4.29

Huanglongbing. See 'Candidatus Liberibacter asiaticus'

Hubbert, L., S4.75

Huber, L., S4.142

Hüberli, D., 1069

Hückelhoven, R., 1214

Hudler, G., S4.178

Hudson, M., S4.27

Huerta, A. I., S4.162

Huerta-Espino, J., 470, 872

Huffman, V., S4.165

Hughes, G., 9

Hughes, M. A., S4.61

Hughes, T. J., S4.90, S4.156

Huguet-Tapia, J., S4.17, S4.62, S4.78, S4.158

Hui-Juan, C., S4.62

Hulbert, S., S4.115

Hulbert, S. H., S4.98, S4.154

Hult, M., S4.113

Hulvey, J., S1.6

Hundie, B., 917

Hunger, R., S4.52

Hunger, R. M., S2.4

Hur, J., S4.72

Hurd, K. M., S2.5

Hussey, R. S., 1362

Hutin, M., S4.37

Hwang, H.-H., 160

Hwang, I., S4.56, S4.83, S4.108

Hwang, S. F., S4.26, S4.51, S4.52, S4.62

Hwang, S.-F., 764

Hypersensitive reaction: effect on Xanthomonas campestris pv. vitians infection in lettuce, 316

Ibrahim, N. F., S4.156

Idowu, J., S4.123

Idris, A. M., S4.7

Ijumba, J. N., 646

Ikerd, J. L., S4.75

Ilaoa, E. M., S4.9

Impullitti, A., S4.66

Independent action hypothesis: testing of, 18

Inderbitzin, P., 662

Information graphs: for binary predictors, 9

Ingel, B. M., S4.62

Inoue, Y., 1568,

Iorizzo, M., 1131

Ipomoea batatas: resistance to Ditylenchus destructor infection by the expression of siRNAs targeting unc-15, 1458

Iqbal, M. J., S4.159

Irby, J. T., S4.88

Iriarte, G., S1.6

Irwin, C., S4.62

Isack, Y., S4.97

Isard, S. A., 905, 947, S4.62

Ishii, H., S4.63

Ishimaru, C. A., S4.93

Isikber, A. A., S4.22

Islam, K. T., 1601, S4.63

Islam, M. M., S4.63

Islam, M. S., 825, S4.63, S4.128

Islas, C., 758

Isocitrate lyase gene: knock-down in root-knot

nematode reduces reproduction, 628

Israeli, O., 1059

Ito, S., 525

Ivors, K., S4.25
Jabaji, S., 1334, S4.130

Jackson, C. S., S2.5

Jackson, E. W., 239

Jackson, V., S4.69

Jacobs, J., S4.19, S4.102

Jacobs, J. L., 378, S4.146, S4.173

Jacobs, J. M., S4.64

Jacobson, D. K., S4.64

Jacoby, T. P., S2.6

Jacques, M. A., S4.31

Jacquot, E., 1408

Jagdale, G. B., S4.102

Jäger, K., 797

Jahn, M., S4.180

Jaime, R., S4.8, S4.64

Jain, M., S4.44, S4.64

Jalan, N., S4.157

Jameson, A., S4.60

Jan, F. J., S4.133

Jancewicz, A. L., S4.85

Jang, C., S4.56, S4.83, S4.108

Janisiewicz, W. J., S4.64

Jankovics, T., 797

Jansky, S. H., 1198, S4.6, S4.46

Jaouannet, M., S4.80

Jardine, D., S4.173

Jardini, T., S4.101

Jardini, T. M., S4.64

Jarosz, A. M., S4.93

Jarugula, S., S4.65

Jatroph mosaic virus: characterization

of strains from the Dominican

Republic, 141

Jayanth, M., S4.65

Jedryczka, M., 895

Jenkins, D. A., S4.156

Jenkins, D. M., S4.76

Jenkins, J. N., S4.145

Jensen, S., S4.130

Jeon, Y., S4.28, S4.72, S4.79

Jeong, T., S4.69

Jeremiah, S. C., 646

Jessup, K. E., 621

Jhala, R., S4.41

Ji, P., S2.8, S2.13, S4.13

Jia, H., S4.38, S4.65

Jia, Y., S4.145

Jiang, H., S1.7, S4.65, S4.94

Jiang, J., S4.28, S4.83, S4.180

Jiang, N., S4.65

Jiang, T., S4.154

Jiang, Z., S4.81

Jifon, J. L., S4.5, S4.27

Jiménez-Gasco, M., S4.12, S4.87

Jin, L., S4.144

Jin, Y., 470, 477, 872, 917, S4.65

Jing, L., S4.100

Jo, Y. K., S4.160

Jochum, C. C., 1146

John, T., S4.176

Johnson, A., S4.37, S4.66

Johnson, A. B., S2.12

Johnson, B., S4.66

Johnson, C. P., 863

Johnson, D., S4.46

Johnson, D. A., 966

Johnson, D. L., S4.68

Johnson, E. G., S4.66, S4.150

Johnson, G. C., S3.1, S3.3, S3.4, S4.180

Johnson, J., 917

Johnson, K., S4.57

Johnson, R., S4.34

Johnson, S. B., 966

Johnson, W., S4.45

Johnston, C. M., S4.116

Johnston, J., S4.54

Jones, B. J., S4.38

Jones, D., S4.10

Jones, J., S2.4
Jones, J. B., S2.8, S2.10, S4.101, S4.110, S4.127, S4.132, S4.157

Jones, J. G., S3.1, S3.3

Jones, L. A., S1.5, S4.66

Jones, M. W., 956

Jones, R., S4.66

Jordahl, J. G., 990

Jordan, B. S., S2.6

Jordan, M., S4.107

Jordan, R., S4.67

Jordan, S., S4.37

Jordan, W. J., S2.3

Jorge-Júnior, A., 805

Joseph, L., 1545, S4.129

Joubert, V., S4.79

Ju, H. K., S4.83

Juarez-Lopez, G., S4.95

Judelson, H., S4.67

Judelson, H. S., 966, 1594

Judge, C., S4.67

Juglans regia: environmental and pathogenic factors inducing brown apical necrosis on fruit, 1427

Jumpponen, A., S4.113

Jung, G., S1.5, S4.6

Junk, J., S4.40

Jurick, W., S4.67

Jurick, W., II, S4.64

Jurick, W. M., S4.87

Juruena, M. H., S4.67

Justesen, A. F., 917

Kabbage, M., S4.116, S4.148

Kachapulula, P., S4.68

Kaczmarek, J., 895

Kaczmarek, M., 895

Kadooka, C., S4.38, S4.140

Kadooka, C. Y., S4.123, S4.124

Kagda, M., S4.67

Kakaire, S., S4.68

Kalia, B., S4.68

Kalih, R., 334

Kalischuk, M. L., S4.68

Kamiya, K., S4.38

Kamo, K., S4.142

Kamvar, Z. N., 684, 982, S4.68

Kanaskie, A., 937

Kanaskie, A. M., 982, S4.68

Kandel, P. P., S2.2, S2.6

Kandel, Y. R., 1601, S4.69

Kanematsu, S., S4.157

Kang, H. J., S4.69

Kang, Z., S4.69

Kang, Z. S., S4.61

Kaplan, J., S4.13

Karakkat, B., S4.69

Karasev, A., S4.34

Karasev, A. V., 1487, S4.29, S4.42

Kariuki, G., 350

Kariyawasam, G., S4.69

Kartanos, V., S4.58, S4.137

Karunakaran, C., S4.77

Kasele, S., 646

Kassem, M. A., 1389

Kasson, M. T., S4.148

Katsoudas, E. J., S4.138

Kaur, G., S4.69

Kaur, J., S4.70

Kaur, N., S4.70, S4.124

Kawai, Y., S3.3

Kawashima, T., S4.70

Kawchuk, L. M., S4.68

Kc, A. N., S2.6, S4.70

Keel, C., S4.90

Kegley, A., S4.83

Kehinde, I. A., S4.4

Keinath, A. P., S4.100

Kella, A. M., S4.138

Kellenberger, S., S4.90 
Keller, K. E., S4.36, S4.60

Keller, N., S4.163

Kellerman, M., S4.71

Kelly, H. M., S2.3, S4.30, S4.71

Kelly, H. Y., 905

Kema, G., S4.105

Kema, G. H., S4.8, S4.166

Kemerait, R., S2.1, S2.12, S4.134, S4.154

Kemerait, R. C., S2.3, S2.13, S4.175

Kennedy, A. H., S4.71

Kennelly, M., S4.113

Kenyon, L., S4.139

Keremane, M. L., S4.71, S4.115

Kern, K. A., S4.51

Kerns, J. P., S4.53

Kerrigan, J., S4.23

Ketelaar, T., 419

Khalilian, A., 674

Khan, A. A., S4.71

Khan, M. F. R., S4.71

Khatri, N., S4.14

Khera, P., S2.3, S2.11, S4.175

Khiutti, A., 1198

Kianian, S. F., S4.6

Kibler, A., S4.119

Kiewnick, S., 1245

Kiggundu, A., 956

Kiirika, L. M., S4.72

Kikkert, J. R., S4.51

Kilian, A., S4.105

Killinger, K., S4.167

Killiny, N., S4.72, S4.171

Kim, B., 1183

Kim, B. S., S4.72

Kim, H. G., S4.108

Kim, H. S., S4.73

Kim, J., S4.29, S4.56, S4.73, S4.83

Kim, J. K., S4.108

Kim, J.-K., S4.125

Kim, K. H., S4.155

Kim, K. S., S4.72

Kim, M. S., S4.16, S4.132

Kim, N., S4.56, S4.83

Kim, N. G., S4.108

Kim, S., S4.73

Kim, T., S4.69

Kim, Y., S4.69

Kim, Y. K., S4.168

Kim, Y. S., S4.72

Kimunye, J. N., 956

Kinard, G. R., S4.135

King, J., S3.1

King, J. G., S4.43, S4.73

Kinsinger, N., S4.116

Kirk, W. W., S4.120

Kirkpatrick, S., S4.58

Kirkpatrick, T., S2.4

Kirkpatrick, T. L., S2.10

Kirsch, M., S4.50

Kiss, L., 797

Kistler, H. C., 1466, S4.163

Kitchen, J. L., S4.73

Kiyuna, J., S4.32

Klaassen, V., S4.8

Kleczewski, N. M., S3.3

Klee, S., S3.3, S4.73

Klein, J. M., S4.73

Klepadlo, M., S4.73, S4.74

Klessig, D. F., S4.88

Kline, L. M., S4.20

Klittich, C. J., S4.34

Kloepper, J. W., S2.7, S2.13, S4.84, S4.151

Klomp, A., S4.91

Klopfenstein, N., S4.16

Klopfenstein, N. B., S4.132

Klos, K. L., S4.126

Klosterman, S. J., 80, 852, S4.77, S4.82, S4.133,

S4.169, S4.176

Kluepfel, D. A., S4.65
Knaus, B. J., S4.134, S4.172

Kness, A. A., S3.4, S4.180

Knight, L. M., S4.15

Knoll, J. E., 1522

Knox, G. W., S4.10

Knut, S. A., S4.9

Kobayashi, D. Y., S3.2

Kobayashi-Leonel, R., S4.74

Koch, A., S4.74, S4.88

Koch, P., S4.69

Kocsis, M., 1104

Koebnik, R., S4.37, S4.64

Koehler, A., S2.6, S4.74, S4.84

Kogel, K. H., S4.74, S4.88

Kohn, J. S., S4.138

Koike, S. T., 80, 638, S4.77, S4.89, S4.109, S4.176

Koita, A., S4.37

Koita, O., S4.136, S4.149

Kokalis-Burelle, N., S4.26, S4.60, S4.120

Koladia, V. M., S4.74

Kolawole, O. O., S4.74

Kolb, F. L., S4.16

Kolmer, J. A., 700

Komáromi, J., 797

Kon, T., 141

Kondo, H., S4.157

Kondo, N., S4.58

Kone, D., S4.149

Konkol, J. L., 433

Kontz, B., S4.103

Koopmann, B., 1137

Korir, N., S4.155

Korir, R. C., S3.4

Korotkin, H. B., S4.20

Koskiniemi, S., S4.162

Kosta, K. L., S4.164, S4.165

Kots, K., 419

Kouadio, L., S4.40

Kouser, T., S4.75

Kousik, C. S., S4.75

Kovacs, L., S4.75

Kovalskaya, N., S4.75

Kovens, M., S4.75

Kraus, C. M., S4.75

Kreis, R. A., S4.76

Kremer, K. R., S4.76

Kreuze, J., S4.59

Kriss, A. B., S4.171

Kroese, D., S4.76

Kronmiller, B., S4.134

Kroschle, J., S4.59

Krstić, B. B., S4.21, S4.76

Kruger, G., S2.2

Kubota, R., S4.76

Kuhn, D. N., S4.111

Kuhn, P., S2.6, S4.4, S4.56, S4.76, S4.135, S4.148

Kuhn, P. J., S4.177

Kuhnem, P. R., 246, 441, 695

Kumar, A., S4.37

Kumar, L., S4.59

Kumar, P., S4.13

Kumar, P. L., 956

Kumar, S., S4.77

Kunjeti, S. G., S4.77

Kunta, M., S4.27, S4.32, S4.85, S4.142

Kuo, C. H., S4.77

Kuo, Y. W., S4.170

Kurjan, J., S4.99

Kurle, J. E., S4.115

Kurowski, C. J., S4.173

Kusia, E., 956

Kwak, Y. S., S4.29, S4.73

Kwenda, S., S4.97

Labavitch, J., S4.62

Labbe, N., S4.20

Labroussa, F., S4.171

Lactuca sativa: downy mildew resistance in ' $\mathrm{La}$

Brillante' conferred by Dm50 gene and multiple
QTL, 1220; effects of host genotype and hypersensitive reaction on Xanthomonas campestris pv. vitians infection, 316 ; transmission of Verticillium dahliae to from spinach, 80

Ladaniya, M. S., 1043

Lagudah, E., 477

Lagudah, E. S., 872

Lahlali, R., S4.77, S4.130

Lahman, M., S4.20

Lai, E. M., S4.162

Lai, E.-M., 160

Lakshman, D., S4.142

Lalancette, N., S4.77

LaMarsh, K., S1.8

Lambert, K. N., S4.90

Lamour, K., S2.3, S4.30, S4.42

LAMP assays: rapid diagnosis of soybean seedling blight and soybean charcoal rot, 1612

Lamppa, R. S., S4.108

Lan, C., 872

Lan, P., S4.24, S4.135

Land, C., S2.7

Lane, S., S4.126, S4.165

Lange, H. W., 169

Langenhan, J., S4.137

Langston, D., S2.3, S2.8, S4.39

Langston, D. B., S2.7

Languasco, L., 325

Lanubile, A., S4.40, S4.78

Lanza, F. E., S4.130

Lapaz, M. I., S4.78

LaPhilliph, P. J., S1.6

Larkin, R. P., S4.78

Larsen, M. M., 982, S4.68

Late blight. See Phytophthora infestans

Latorre, B. A., S4.36

Laurel wilt. See Raffaelea lauricola

Laville, N., S1.3

Lawes, R., 1069

Lawler, T. L., S4.65, S4.78, S4.134

Lawrence, A., S4.2

Lawrence, D., S4.78

Lawrence, D. P., S4.138

Lawrence, K. S., S2.7, S2.12, S4.86, S4.151

Lawson, N., S4.156

Leach, J. E., S4.18, S4.37, S4.138

Leaf gas exchange: in avocado, effect of Raffaelea lauricola infection on, 433; in maize, effect of Stenocarpella macrospora infection on, 26; in rice, effect of Monographella albescens infection on, 180; in rice, effect of silicon on in Pyricularia oryzae infected plants, 738

Leaf oxidative metabolism: in rice, effect of silicon on in Pyricularia oryzae infected plants, 738

Leandro, L. F. S., 1601, S4.1, S4.8, S4.31, S4.69, $\mathrm{S} 4.74$

Lebeau, A., 1529

Lebeda, A., 998

Lebeis, S., S4.173

Leblanc, M. L., S4.78

Leca, A., S4.79

Lecomte, P., S4.138

Leconte, M., S4.142

Lee, D., S4.154

Lee, I. H., S4.28

Lee, I. M., S1.2

Lee, J., S4.132

Lee, J. H., S4.79

Lee, J. K., S4.79

Lee, J. Y., S4.99

Lee, K. D., 1114, 1123

Lee, K. M., S4.155

Lee, R. D., S2.3, S2.13, S4.175

Lee, R. F., S4.71, S4.115

Lee, S., 580, S4.49, S4.79

Lee, S. A., S4.80

Lee, S. H., S4.79

Lee, W. S., S4.127 
Lee, Y. H., 412

Lee, Y. K., S4.99

Lefeuvre, P., S4.64

Legg, J. P., 646, S4.59, S4.98, S4.155

Lehman, B., S4.116

Lehner, M. S., S4.80

Lehnoff, E. A., S4.116

Leisner, S., S4.3

Leite de Oliviera, M., S4.80

LeJeune, J. T., S4.14

Lekuona, H., S4.149

Lelis, F. M. V., S4.80

Leng, Y., 482

Lenoir, C., S4.80

Lenzi, P., S4.80

Leon, A. L., S4.80

Leon, G., 1013, S4.121

Leonardo, L., S4.131

Lerch, E. R., S4.81

Leslie, J. F., S4.21, S4.91, S4.155

Lettuce downy mildew. See Bremia lactucae

Leung, H., S4.18

Leveau, J. H. J., 1036, S4.35, S4.37, S4.53, S4.116

Levesque, C. A., S4.85

Levi, A., S4.136

Levy, J., S4.81

Levy, L., 564

Lewis, K. J., 69

Lewis, P., S4.106

Lewis, R. S., S4.91

Lewis Ivey, M. L., S2.9

Li, A., 840, S4.11, S4.134

Li, C., S4.158, S4.174

Li, D., S4.83

Li, F., S4.24, S4.135

Li, H., S4.82

Li, H. X., S4.48, S4.82

Li, H.-X., 815

Li, J., S4.47, S4.65, S4.81, S4.82

Li, M., S4.81

Li, P., S4.163

Li, R., S4.24, S4.83, S4.106, S4.135

Li, S., S4.25, S4.82

Li, W., S4.81, S4.144

Li, X., 424, S4.63, S4.69, S4.100, S4.152

Li, Y., 1325, S4.81

Li, Z., S4.82, S4.176

Liang, C., S4.82

Liang, J., 1466

Liang, Z., S4.101

Liao, X., 1325

Lichty, J., S4.129

Lim, H. J., S4.83

Lim, H. S., S4.56, S4.83, S4.108

Lim, H.-S., S4.125

Lim, S., S4.125

Lima, E. A., 805

Lin, B., S4.157

Lin, D., S4.12

Lin, H., 825

Lin, J. S., S4.162

Lin, M., S4.145

Lin, S., S4.109

Lin, Y., S4.83

Lin, Y. H., S4.26, S4.28, S4.29, S4.83

Lin, Y. J., S4.83

Lindbo, D. L., S4.53

Lindsey, L. E., S4.85

Ling, K.-S., S4.83, S4.106

Linkmeyer, A., 1214

Linley, R., S4.104

Little, C. R., S4.11, S4.12, S4.21, S4.109

Liu, B., S4.84, S4.109

Liu, F., 588, 1318

Liu, G., 1458

Liu, H., S4.82

Liu, J., S4.31, S4.84

Liu, J. J., S4.83

Liu, K., S2.7, S4.84
Liu, M., S4.85

Liu, S., S4.8, S4.11, S4.96, S4.131

Liu, Y., S3.1

Liu, Y.-T., 160

Liu, Z., S4.69, S4.144, S4.146, S4.147, S4.155

Liu, Z. P., S4.158

Livingston, W., S4.99

Llaca, V., 1362

LNA-based qPCR assays: detection and

identification of Meloidogyne enterolobii, 1245

Lockhart, B. E., 956, S4.56, S4.67

Loeb, G. M., S4.56

Lofgren, L., 1466

Logan, J., S4.55

Lolas, M., S4.36, S4.84

Lolium perenne: silicon-induced systemic defense responses against Magnaporthe oryzae infection, 748; STEMRUST_G model, 35, 45

Lombard, K., S4.99

Lommel, S. A., S4.53

Long, A., S4.100

Lookabaugh, E., S4.84, S4.85

Loop-mediated isothermal amplification. See LAMP assays

Loper, J., S4.73, S4.132

Loper, J. E., S4.110

Lopes, C. A., S4.123

Lopez, S., S4.46

Lopez-Escudero, F. J., S4.106

Lopez-Herandez, J. F., S4.107

Lopez-Nicora, H. D., S4.85

Lopez-Ramos, C., S4.60

Lopez-Vera, E. E., 470

Loria, R., S4.62, S4.78, S4.158

Lourenço-Tessutti, I. T., 628

Louws, F., S4.62

Louws, F. J., S4.3

Louzada, E. S., S4.85, S4.142

Low, D. A., S4.162

Lowe, C., S4.85

Lowe, T. M., S4.85

Lowery, T., 1373, S4.112

Loyd, A., S4.154

Lozovaya, V. V., S4.109

Lu, A. Y., 863

Lu, C., 1612

Lu, S., S2.12, S4.85, S4.86, S4.148

Luambana, N., 350

Luangkhot, J. A., S4.86

Lubell, M., S4.13

Lucas, H., S4.148

Lucks, J., S4.137

Lujan, M., S4.165

Lujan, P., S4.69, S4.86, S4.122, S4.123

Luke, E. M., S3.3, S4.126

Lundberg, D., S4.173

Lundquist, J. E., S4.16

Lunos, A., S4.86, S4.110

Luo, C., S4.130

Luo, L., S4.47, S4.65, S4.82

Luo, W., S4.86, S4.87

Luo, Y., S4.106

Lupien, S. L., S4.38

Luque, A., S4.123

Lusley, P., S4.142

Luster, D., S4.65

Luster, D. G., 91, 917, S3.3, S3.5, S4.43

Luteovirus: detection of a new virus in imported nectarine trees, 840

Luther, T., S4.99

Lv, Q., S4.65

Lygin, A. V., S2.2, S4.109, S4.128

Lysobacter enzymogenes: PilG regulation of twitching motility and antifungal antibiotic biosynthesis in, 1318

Ma, B., S4.121

Ma, H., 771

Ma, L. J., 885, S4.54, S4.61, S4.158, S4.175, S4.176
Ma, L. S., S4.162

Ma, P., 1458

Ma, Y., 771

Ma, Z., 1466, S4.174

Mabon, R., S4.88

Macarisin, D., S4.87

Macarisin, O., S4.87

MacArthur, J. L., S4.162

Maccaferri, M., S4.8

MacCoss, M. J., S4.34

Macedo, M., S4.87, S4.143

Machado, M. A., 518

Maciel, J. L. N., 284

MacIntosh, G., S4.1

MacIntyre, A., S4.138

Mackie, D. C., S4.28

Macleod, W. J., 1069

Macluravirus, 1155

Macrophomina phaseolina: diagnosis in soybean using LAMP assays, 1612

Macrospora leaf spot. See Stenocarpella macrospora

Madden, L. V., 210, 295, 1174, 1400, S4.96, S4.97, S4.122, S4.171

Maderias, A., 885

Maffia, L. A., S4.18, S4.25

Magdama, F. A., S4.87

Magill, C., S4.169

Magnaporthe oryzae: population structure, pathogenicity, and mating type distribution of isolates from East Africa, 1137; QoI resistance in Brazilian populations, 284; silicon-induced systemic defense responses in perennial ryegrass, 748

Mahaffee, W., S4.136

Mahaffee, W. F., S4.142

Maharaj, N., S4.37

Maharaj, N. N., S4.53

Mahillon, M., S4.87

Mahoney, J., S4.97

Mahuku, G., 956

Maia, A. H. N., 307

Maier, T. R., 1362

Maiss, E., S4.37

Maize lethal necrosis: emerging threat in

Sub-Saharan Africa, 956

Maize rayado fino virus: infectious, from cloned cDNA, 833

Malapi-Wight, M., S4.88, S4.124

Malik, A. U., S4.117

Mallory-Smith, C., S4.30, S4.103

Mallowa, S., S4.155

Mallowa, S. O., 1080

Malmstrom, C. M., S4.39

Malossini, U., 555

Malus $\times$ domestica: effect of Brassica seed meal soil amendments on production, 460

Malvick, D., S4.131

Malvick, D. K., 1601, S4.93

Malvick, D. M., S4.44, S4.115, S4.173

Mamidi, S., 500

Mamo, B. E., 99

Mandel, M. A., S4.127

Mangifera indica: reassessment of recently

described species of Ceratocystis fimbriata on, 1229

Mann, R., S4.8, S4.96, S4.131

Manohar, M., S4.88

Manolii, V. P., 764, S4.51

Manosalva, P., S4.88, S4.92

Mansour, W. J., S4.88

Manter, D., S1.5, S4.6

Mantooth, W., S4.94

Manulis-Sasson, S., S4.49

Maple bark biochar: effect on Rhizoctonia solani metabolism and damping-off severity, 1334

Marais, A., 135, 1155

Marek, S., S4.41, S4.66, S4.105

Marek, S. M., 656, 684, S4.97, S4.109

Marek, T., S4.167 
Marett, C. C., S4.140

Margaria, P., S4.88

Mariette, N., S4.88

Markell, S., S4.50

Markell, S. G., 990

Marks, M. E., S4.89

Marocco, A., S4.78

Marois, J. J., 905

Marquez-Karry, R. G., S4.156

Marra, R. E., S1.7

Marroquin-Guzman, M., S4.89

Marshall, S. H., 388

Martin, C., S4.41, S4.89

Martin, D. P., 716

Martin, F. N., 265, S4.57, S4.77, S4.89, S4.96, S4.132, S4.165, S4.168, S4.169

Martin, G. B., S4.75

Martin, K., S4.60

Martin, K. M., S4.89

Martin, M., S4.118

Martin, R. R., S4.36, S4.60

Martin, S. B., S4.23

Martinez-Cisneros, B., S4.95

Martins-de-Sa, D., 628

Marzano, S. Y. L., S4.90

Mascher, F., S4.89, S4.90

Mascia, T., 1155

Mason, R. E., 1114

Masson, P. H., S4.85

Mater, Y. J., S4.90

Mathew, F., S4.42, S4.50, S4.103

Mathew, F. M., 990, S4.90

Matson, M. E. H., 1594

Matsukura, K., 550

Matsumura, M., 550

Matsushita, Y., S4.9

Matthiesen, R., S4.90

Matusinsky, P., 895

Mauch-Mani, B., S4.89

Maul, J. E., 1325

Mauleon, R., S4.138

Maurer, H. P., 334

Maurhofer, M., 199

May, S. R., S4.109

Mayorquin, J., S4.92

Mayorquin, J. S., S4.91, S4.180

Mays, D., S4.91

Mazzola, M., 460, S4.4, S4.59, S4.117, S4.127, S4.145, S4.159

Mbofung, G., S4.91

McAninch, G., S4.165

McAvoy, E., 388, S4.156

McBride, S. A., S2.7

McCallum, B., S4.107

McCallum, B. D., 728

McCarty, L., S4.23

McClung, A. M., S4.159

McClure, A., S2.2

McClure, A. M., S4.94

McCluskey, K., S4.91

McCollum, G., S4.86

McCollum, G. T., S4.115

McCook, S., 1164

McCorkle, K., S4.84

McCorkle, K. L., S4.91

McCreight, J. D., S4.13

McCullough, M., S4.91, S4.102

McDonald, B. A., 284, 1475

McDonald, M. R., S4.51, S4.52, S4.92

McDuffee, D., S4.102

McFarland, K. A., S4.77

McGhee, G. C., 1302

McGrann, G. R. D., 895

McGrath, M. T., 388, 885, 966, S1.8, S4.92

McGregor, L., S4.130

McHale, L. K., S4.49

McInroy, J. A., S2.7, S4.84, S4.151

McKay, A., 1069

McKee, B., S4.92
McKeever, K., S4.93

McKemy, J. M., S3.3, S4.126

McLaren, D. L., S4.26

McLeod, A., S4.71

McMahon, M., S4.43

McMahon, M. B., S3.3

McManus, P., S4.112, S4.137

McMechan, A. J., 1496

McNally, R. R., S4.93

McNellis, T., S3.3, S4.73, S4.93, S4.116

McNew, D., S4.169

McNew, D. L., S4.151

McOwen, N., S4.158

McRoberts, N., 9, S4.8, S4.50, S4.176

McTavish, C. K., S4.93

Mechan Llontop, M. E., S4.93

Medeiros, F. H., S4.128

Medeiros Del Ponte, E., S4.33

Medina, R., S4.46, S4.94

Medrano, E. G., S4.94

Mefenoxam resistance: acquired in Phytophthora infestans, 342

Mehl, H. K., S4.19

Mehl, H. L., S4.94

Mehra, L., S4.94

Mehra, L. K., 1417

Mehrvar, M., S4.87

Meijer, H. J. G., 419

Meinhardt, S., S4.6

Meinholz, K., S4.36

Melby, D., S4.80

Melcher, U. K., S4.39

Melgarejo, T., S4.143

Melgarejo, T. A., 141

Mellbye, M. E., 35

Mellinger, H. C., 388, S4.11

Meloidogyne spp.: mitochondrial haplotype-based identification, 350; resistance in Coffea canephora, 805

$-M$. enterolobii: LNA-based qPCR assays for detection and identification, 1245

$-M$. incognita: inheritance and identification of a major QTL for resistance in sweet sorghum, 1522; knock-down of HSP90 and isocitrate lyase gene expression reduces reproduction in, 628

Melouk, H., S4.41

Melzer, M., S4.80

Melzer, M. J., 1013, S4.29, S4.121, S4.157

Menalled, F., S4.53

Menalled, F. D., S4.116

Menard, R. D., S4.45

Mendes, O., S4.105, S4.166

Mendez, R., S4.36, S4.84

Mendoza, A., S4.81

Meng, Q., S4.65, S4.94

Meng, Y., S4.158

Mengistu, A., S4.94

Menzies, J., S4.95

Mergoum, M., S4.69

Mertely, J., S4.95

Metagenomics: of plant viruses, review article, 716; proposal to adopt in post-entry quarantine, 840

Metalaxyl resistance: in Phytophthora infestans, role of RPA190 alleles in, 1594

Meyer, M., 1288

Meyer, S., S4.50

Meyer, S. L. F., S3.1

Meyer, S. M., 990

Meyers, E., S2.7

Mezzalama, M., S4.95

Mgonja, E., S4.95

Mhora, T. T., S4.95

Mian, R., S4.49

Miano, D. W., S4.98, S4.155

Micallef, S. A., S3.4

Michael, J. L., S4.21

Michailides, T., S4.97

Michailides, T. J., S4.106

Michalides, B., S4.100
Michel, A. P., S4.49

Michelmore, R., S4.77, S4.169

Michelmore, R. W., 1220

Micijevic, A., S4.103

Miedaner, T., 334

Mihelich, N., S4.96

Miklas, P., S4.108

MILDEW LOCUS O: mutation of does not affect resistance to grain infections with Fusarium spp. and Ramularia collo-cygni, 1214

Miles, T. D., 265, S4.57, S4.96, S4.165

Milgroom, M. G., 370, 1250

Milies, L. A., S4.97

Miller, B., S2.4

Miller, J. C., S4.81

Miller, N. F., S4.96

Miller, S. A., 126, S4.13, S4.14, S4.63, S4.136, S4.143

Miller, T., S4.144, S4.154

Miller, Z., S4.112

Miller, Z. J., S4.116

Millett, B. P., 1131

Mills, K., S4.96

Milojević, K. N., S4.21, S4.76

Milus, E. A., 1114, 1123

Minicka, J., 1270

Minutillo, S. A., 1155

Mirkov, E. T., S4.5

Misner, I., S4.142

Mitchell, T., S4.120, S4.135

Mitchell, T. K., 656, S4.24, S4.142

Mitchell, T. R., S4.10, S4.16

Mitchum, M. G., 1362

Mitochondrial haplotypes: identification of

Meloidogyne spp. with, 350

Miyan, S., 1069

Miyao, E., S4.37

Mizubuti, E., S4.123

Mizubuti, E. S. G., S4.80

Mkamilo, G. S., 646

Mlotshwa, S., S4.162

Mohabbatizadeh, M., S4.57

Mohamed Nor, N. M., S4.155, S4.156

Mohammad, A., S4.96

Mohd, M., S4.156

Mohr, J., S4.97

Moktan, K., S1.5

Moleleki, L. N., S4.97, S4.105

Molina, A. B., S4.67, S4.166

Mollov, D., S4.56, S4.67, S4.135

Moncolva-Santana, C., S1.3

Moncrief, I. R., S4.97

Monfort, S., S2.10

Monilinia spp.

-M. fructicola: fine-scale genetic structure in peach tree canopies, 542

-M. vaccinii-corymbosi: population structure in the U.S., 533

Monis, J., S4.97

Monographella albescens: effects of infection on leaf gas exchange and chlorophyll $a$

fluorescence in rice, 180, cover photo: February

Monoterpenes: effect on spore germination and growth in Fusarium circinatum, 119

Monshausen, G., S3.3, S4.73

Montarry, J., S4.88

Monteil, C. L., S4.93, S4.143

Montesdeoca, F., S4.59

Montesdeoca, L., S4.59

Moon, D. E., 1114

Moore, W. F., S4.3

Moorhead, J., S4.167

Moraes, W. B., S4.97

Mora-Herrera, M. E., S4.48

Moral, J., S4.97, S4.106, S4.120, S4.141

Morales-Cruz, A., S4.47

Morelli, M., 555

Moreno, A., S4.32

Morgan, G. D., S2.12 
Morgan, J. K., 608

Morgan, M., S4.90

Morozov, M., S4.49

Morris, K. A., S2.7, S4.98

Morris, T. J., 126

Mortensen, C. N., S4.82

Mosquera, G., S4.18

Motghare, M., 1043

Mou, Z., S4.110

Moury, B., S4.22

Moustaid-Moussa, N., S4.20

Mowery, P., S4.57

Moya, A., S4.109

Moya-Elizondo, E. A., S4.98

Mueller, D. B., S4.173

Mueller, D. S., 1601, S4.8, S4.69, S4.74, S4.90

Mueller, J. D., 674

Mueller, T. A., S4.140

Mueth, N., S4.154

Mueth, N. A., S4.98

Muhanna, M. M., 646

Mukanga, M., S4.63, S4.68

Mukherjee, K., 1043

Mukuma, C., S4.131

Mulchandani, A., S4.107

Mulenga, R. M., S4.98

Mullis, S. W., S2.9

Munck, I., S4.99, S4.177

Munir, M., S2.7

Munkvold, G. P., S4.31, S4.40, S4.78, S4.147

Munkvold, K. R., S4.75

Muñoz, J. E., S1.6

Munoz, M. E., S4.27

Munyaneza, J. E., 1573

Muppirala, U. K., S4.78

Muramoto, J., S4.59

Murata, M. M., S4.99

Murphy, A. M., S4.3

Murphy, C. A., 91

Murphy, J. A., S4.180

Murray, T. D., S4.126

Musa spp.: Fusarium wilt, review article, cover photo: December, 1512

Musil, K. M., S4.99

Mustafayev, E., 135

Musungu, B. M., S4.134

Mutai, C., S4.49

Mutiga, S., S4.120

Mutiga, S. K., 1250

Mwanga, R. O., S4.179

Mycology: differences in virulence and pathogenicity of Verticillium longisporum lineages, 662; effects of resin and monoterpenes on spore germination and growth in Fusarium circinatum, 119; Fusarium oxysporum f. sp. cepae genetic and pathogenic variability in onion and Welsh onion, 525; interactions of Phytophthora capsici with resistant and susceptible pepper roots and stems, 1355; observation of ascosporic infection in Blumeria graminis f. sp. tritici, 797, cover photo: June; reassessment of recently described species of Ceratocystis fimbriata on Mangifera indica 1229; role of RPA190 alleles in metalaxyl resistance in Phytophthora infestans, 1594

Myers, G. T., S4.64

Myers, J. R., 1487, S4.42

Myers, K., 342, S4.33, S4.104

Myers, K. L., 966

Myers, R., S4.129

Mysore, K., S4.50

Mysore, K. S., 580

Myung, I. S., S4.99

$\mathrm{Na}, \mathrm{F}, \mathrm{S} 4.180$

Naegele, R., S4.99

Naegele, R. P., 110

Nagaraju, A., S4.61

Nagata, A., S4.143
Nagendran, R., 412

Nagl, N., S4.21

Naidu, R. A., S4.143

Naka, T., S4.9

Nakahara, K., 525

Nakamura, C. V., S4.51

Nakhla, M. K., 564, S4.15, S4.22, S4.144, S4.146

Nam, S. Y., S4.69

Nanami, D. S., S4.51

Nancarrow, N., S4.139

Narayanaswamy, B., S4.100

Nargi, F., S4.146

Narouei Khandan, H. A., S4.100, S4.141

Nascimento, K. J., S4.34

Natarajan, B., 947

Natural, M. P., S4.67

Natwick, E. T., S4.13

Navarro, K., S4.46

Navi, S. S., S4.100

Ndukwe, P., S4.119

Ndunguru, J., S4.98

Ndyetabula, I. L., 646

Neate, S. M., S4.137

Neher, O. T., S4.21, S4.56

Nehring, J., S4.50

Nelson, B., S4.29

Nelson, B. D., S4.90, S4.173

Nelson, R. J., 1250

Nelson, S., 1347

Nelson, S. C., S4.110

Nematology: factors affecting spatial distribution of reniform nematode in cotton, 674;

LNA-based qPCR assays for detection and identification of Meloidogyne enterolobii, 1245 ; mitochondrial haplotype-based identification of Meloidogyne spp., 350; new candidate effectors of Heterodera glycines produced in esophageal gland cells, 1362, cover photo: October; resistance of Coffea canephora to Meloidogyne spp., 805; resistance to Ditylenchus destructor infection in sweet potato by the expression of siRNAs targeting unc-15, 1458

Nemchinov, L. G., S4.100, S4.113

Nester, S. E., 91, S3.3, S4.18

Neufeld, K. N., S2.8, S4.100

Neupane, A., 500, 509

Neupane, K., S4.80

Newark, M. J., S2.8, S3.4

Newberry, E. A., S2.8, S4.101

Newcomb, M., 917, S4.65

Newfield, M., S4.30

Newman, B., S4.124

Newman, S., S4.141

Next-generation sequencing: versus biological indexing for detection of grapevine viral pathogens, 758

$\mathrm{Ng}$, J. C., S4.27, S4.170

Nguyen, B., S4.135

Nguyen, K., S4.101

Nguyen, T. L., S4.121

$\mathrm{Ni}, \mathrm{X}, \mathrm{S} 2.13$

Niblack, T. L., S4.85, S4.128

Niblett, C. L., 956

Nichols, B., S4.64

Nichols, R. L., S4.31, S4.84

Nicole, M., 805

Nicoletta, K. R., S4.101

Nicolli, C. P., 246

Nie, X., S4.101

Niemira, B. A., S4.168

Nieto-Angle, D., S4.48

Nikolaeva, O. V., S4.29

Nikolić, D. M., S4.21, S4.76

Nischwitz, C., S2.9, S4.91, S4.102

Nissan, G., S4.49

Nita, M., S4.104

Niu, C., S4.102

Njiti, V., S4.158

Njuguna, J., S4.49
Nocchi, P. T., S4.51

Noe, J. P., S4.102

Noel, N., S4.24

Noel, Z. A., S4.102

Nong, H., S4.81

Nonhost resistance: versus host resistance, review article, 580

Noon, J. B., 1362

Nordgaard, J., 1573

Noreen, R., S4.102

Norman, D., 1183, S4.6

Norman, D. J., S4.17, S4.71

Nouri, S., S4.103, S4.170

Novakazi, F., 662

Novy, R., 1573

Nunes, W. M. C., S4.51

Nusayr, T., S4.103

Nyasani, J. O., 956

Obert, D. E., 239

Oblinger, B. W., S4.103

Oblufolaji, D. B., S4.104

Ocamb, C. M., S4.30, S4.103

Ochoa, O., S4.169

Ochoa, O. E., 1220

Ochoa, R., 1013, S4.67, S4.121

Ochoa-Corona, F., S4.67, S4.124

Ocimum spp.: basil downy mildew resistance in, 778

-O. basilicum: effect of shoot potassium

concentration on gray mold in, 1059

Ocsoy, I., S4.132

Ogawa, M., S3.3

O'Gorman, D., S4.140

O'Gorman, D. T., 1373

Oh, D. H., S2.5, S4.127

Oh, S. K., S4.103

Oh, Y., S4.176

O'Hara, C. O., S2.4

Ohbu, M., S4.70

Ojeda, H., S4.138

Ojiambo, P., S4.114, S4.137, S4.169

Ojiambo, P. S., 998, 1417, S2.8, S4.94, S4.100

Okagaki, L H., S4.176

Okello, P., S4.103

Okuda, M., 550

Oladipupo, O. A., S4.4

Olaya, G., S4.104

O'Leary, M. L., S4.104

Oliva, J., 1191

Oliva, R., S4.37

Oliveira, L. S. S., 1229

Oliveira, M. S., S4.104

Oliver, C., S4.104

Oliver, J. E., 855

Olivera, P., 917, S4.65

Olsen, N., S4.124

Olsen, R. T., S4.54

Olson, J., S4.67

Olson, T., S4.103

Olsson, C. H. B., 1191

Omar, A. A., S4.99

Onaga, G., 1137

O'Neal, S., S4.42

O'Neill, P. M., 786, S4.47

Ong, K., S4.105

Ong, K. L., S2.1, S2.9

Onkendi, E. M., S4.105

Onofre, R. B., S4.105

Onuki, M., 550

Oosterhof, J., S4.14

Ophiosphaerella korrae: infection and

colonization of bermudagrasses, 656

Opperman, C. H., S4.53

Ordaz, N. A., S4.105

Ordonez, N. I., S4.166

Ordoñez-Morales, K. C., S4.141

Ordonez Roman, N. I., S4.105

Orobovic, V., S4.93 
Oropeza-Navarro, R., S4.128

Orquera, G., S4.66, S4.105, S4.109

Orshinsky, A., S3.2, S4.60

Ortega-Beltran, A., S4.106

Oryza sativa: effects of Monographella albescens infection on leaf gas exchange and chlorophyll $a$ fluorescence, 180, cover photo: February; effects of silicon on gas exchange and oxidative metabolism in leaves infected by Pyricularia oryzae, 738; emergence of Rhizoctonia solani AG-1 IA in South America via a host shift from, 1475

Osei, M. K., S4.90

Osman, F., S4.33, S4.135

Osnaya-González, M. L., S1.2

Osorno, J. M., S4.108

Ospina-Giraldo, M. D., S4.106

Osterbauer, N. K., S4.165

Osti, S., S4.109

Ostos, E., S4.106

Ostrofsky, W., S4.99

O’Sullivan, D., S4.95

Ota, Y., S4.132

Otero-Colina, G., 564, 1013, S4.121

Oudemans, P., S4.24

Oudemans, P. V., S1.7, S1.8

Ouimette, D., S4.106

Overstreet, C., S2.4

Ownley, B. H., S4.20, S4.30, S4.127

Ozkan, H. E., S4.97

Padgett, B., S2.9

Padmanabhan, C., S4.106

Paetzold, L., 189

Paez, C. A., S4.107

Pagan, C., 350

Pagliaccia, D., S4.107, S4.135

Pain, A., S4.142

Palmateer, A. J., S4.133

Palmer, C. L., 91, S3.3, S4.18

Palomares-Rius, J. E., S4.133

Palukaitis, P., S4.155

Pan, H. R., S4.107

Pandey, M. K., S2.11

Panicum mosaic virus: and its satellite virus infection of switchgrass breeding plots, 1146, cover photo: August

Paniucm virgatum: Panicum mosaic virus and its satellite virus infection of breeding plots, 1146, cover photo: August

Panwar, V., S4.107

Paolinelli-Alfonso, M., S4.107

Pap, D., S4.75

Pappu, H., S4.115, S4.130

Pappu, H. R., 956

Parastagonospora nodorum: effects of wheat residue on severity and yield in winter wheat, 1417

Pardo, J. M., S1.3, S1.5

Pardo Garcia, J. M., S1.6

Parent, L. C., S4.57

Paret, M. L., S2.8, S4.10, S4.67, S4.101, S4.132

Pargas, R., S4.124

Parish, A., S4.106

Park, C. H., S4.83

Park, E., S4.174

Park, J. H., S4.79

Park, J. S., S4.108

Park, M., S4.155

Park, R., 490

Park, W. J., S4.23

Parke, J., S4.46

Parke, J. L., S4.110

Parker, J., S2.2

Parker, M., S4.59

Parks, K., S4.34

Parks, R., S2.7

Pasche, J., S4.160

Pasche, J. S., S4.108, S4.153
Pastalka, T., S4.108, S4.165

Pastor-Corrales, M., S4.108

Patel, J. S., S2.8, S4.108

Pathogen mode of action: independent action

hypothesis, 18

Patpour, M., 917

Patzoldt, M. E., S4.95

Paugh, K. R., S4.108

Paul, K., S4.104

Paul, P. A., 210, 295, 1080, S4.85, S4.96, S4.97,

S4.118, S4.122, S4.126, S4.128, S4.171

Paula Júnior, T. J., S4.80

Paulitz, T., S4.154

Paulitz, T. C., S4.9

Pavel, J., S4.109

Paveley, N. D., S4.73

Pavlu, J. C., S4.109

Pawlowski, M. L., S4.109

Payet, A., 1155

Payne, G. A., S4.102

Payton, M., S4.52

Payton, M. E., S2.4

Pearce, T., S4.125

Pecchia, J., S4.58

Pechy-Tarr, M., S4.90

Pedersen, J. F., 786

Pederson, S., S4.153

Pedley, K., S4.35

Pedley, K. F., S4.111, S4.142

Pedrozo, R., S4.109

Peduto Hand, F., S4.109, S4.142

Pegues, M., S2.4

Pel, M. A., 1220

Pena, M. M., S4.162

Pena, R., S4.77

Peña-Olivares, S., S1.2, S1.3

Peñaranda, E. A., S4.71

Peng, G., S4.51, S4.52, S4.62, S4.77, S4.130

Peng, J., S4.109, S4.110

Pensec, F., 1529

Pentimone, I., S4.163

Pepino mosaic virus: alignment-guided

mutagenesis of an attenuated, cross-protective variant, 126

Pereira, D. A. S., 1475

Pereira, J., S4.110

Perennec, S., 1155

Peres, N. A., S4.7, S4.31, S4.45, S4.95, S4.104,

S4.105, S4.125, S4.160, S4.171

Pereyra, S., 895

Pérez Rodriguez, M., S4.97

Perkins, K., S4.149

Perna, N., S4.121

Peronospora belbahrii: discoveries and challenges in control of, 885; resistance in Ocimum species, 778

Perring, T. M., S4.116

Perry, K., S4.137

Perry, K. L., 1026

Persea americana: effects of Raffaelea lauricola

on leaf gas exchange and xylem sap flow, 433

Pertot, I., 199

Pesce, C., S4.64

Pesic-VanEsbroeck, Z., S4.6

Peter, K., S4.24, S4.116

Petersen-Berger, J., S3.4

Peterson, E. K., 937, S4.110

Peterson, G., S4.96, S4.111

Pethybridge, S. J., 358, S4.51, S4.110

Petkar, A., S2.8

Petrizzo, E., S4.102

Pettis, G., S4.62

Pfender, W. F., 35, 45

Pfeufer, E., S4.116

Phakopsora pachyrhizi: history, epidemic

evolution, and model burn-in for a network of annual invasion, 947; meta-analysis of the relationship between severity and crop yield, 307; response to in North America, 905
Pham, H., S4.62

Phannareth, T., S3.4

Phaseolus vulagaris: Bean common mosaic virus isolate able to overcome the $b c-3$ resistance allele in, 1487

Phelan, D. M., S4.15

Phibbs, A., S4.67

Philion, V., S4.79

Philippe Rolshausen, P., S4.154

Philmus, B. J., S4.110

Phomopsis stem canker: reemergence as a threat to sunflower, 990

Phuntumart, V., S4.14

Phyllosphere microbiology: artificial surfaces in, review article, 1036

Phytophthora fruit rot resistance: worldwide evaluation of Capsicum annuиm for, 110

Phytophthora spp.: rapid isothermal amplification assays for detection of, 265

-P. alni: subspecies distribution in southern Sweden, 1191

- P. capsici: inhibition by silver nanoparticles synthesized with aqueous extract of Artemisia absinthium, 1183; interaction with resistant and susceptible pepper roots and stems, 1355; worldwide evaluation of Capsicum anпиит for resistance, 110

-P. infestans: acquired mefenoxam resistance, 342; age-dependent correlation of resistance in tubers with $R B$ transgene transcript levels, 1131; effect of flumorph on F-actin dynamics, 419; effect of temperature on growth and sporulation of clonal lineages US-22, US-23, and US-24, 449; evaluation of BlightPro decision support system for management of, 1545; population structure in a potato germplasm nursery, 771; potential effects of diurnal temperature oscillations on, 230; reasons to consider as a reemerging pathogen, 966; role of RPA190 alleles in metalaxyl resistance in, 1594; testing taxonomic predictivity of foliar and tuber resistance in wild Solanum spp., 1198

- P. parasitica: inhibition by silver nanoparticles synthesized with aqueous extract of Artemisia absinthium, 1183

- $P$. ramorum: detection, diversity, and population dynamics of waterborne populations, 57; spatial and temporal analysis in Oregon, 982; temporal epidemiology in Oregon, 937

Pianzzola, M. J., S4.31, S4.78

Pieck, M., S4.111, S4.142

Pierson, E., S4.81

Pieterse, C. M. J., S4.147

pilG gene, 13108

Pilkington, S., S4.125

Piotrowska, M., 895

Pires, R. P., S4.44

Pirolo, C., 555

Pisani, C., S4.111

Pitino, M., S4.111

Pitman, A. R., 863

Pitman, T., S4.111, S4.170

Pittman, E. W., S4.16

Piya, S., S4.111

Plaisance, A., S4.153

Plant diseases: emerging and re-emerging, focus issue articles on, 852

Plant-fungal interaction modeling, 482, cover photo: April

Plasmodiophora brassicae: characterization of Cr811 gene in pathotype 5, 764

Ploetz, R. C., 433, 1512, S4.38, S4.111, S4.127, S4.166

Plum pox virus: assessing the mismatch between incubation and latent periods, 1408

Podosphaera xanthii race 1W: inheritance of resistance in watermelon, 1446, cover photo: November

Poduch, K., S4.10

Pojoski, T., S4.146 
Pokhrel, S., S4.112

Pol, C., S4.112

Pol, C. I., S4.53

Poland, J., S4.68

Polashock, J., S1.8, S4.112

Poloni, N. M., 1475

Polston, J. E., S4.156

Ponnala, R., S4.10

Poojari, S., S4.112

Poole, G., S4.7

Poole, G. J., 1069

Poole, S., S4.162

Pooler, M. R., S4.54

Population biology: changes in genetic composition of Stagonosporopsis tanaceti in Australian pyrethrum, 358; emergence of Rhizoctonia solani AG-1 IA in South America via a host shift from rice, 1475 ; factors affecting the composition of Fusarium head blight pathogens in a subtropical no-till wheat agroecosystem, 246; genetic diversity and population structure of cucurbit gummy stem blight, 815; mechanisms of azole resistance in Erysiphe necator, 370; molecular polymorphism and phenotypic diversity in Eutypa lata, 255;

Monilinia fructicola fine-scale genetic structure in peach tree canopies, 542; population structure, pathogenicity, and mating type distribution of Magnaporthe oryzae isolates from East Africa, 1137; population structure of Monilinia vaccinii-corymbosi in the U.S., 533; population structure of Pythium irregulare, $P$. ultimum, and $P$. sylvaticum in forest nursery soils, 684; population structure of Xylella fastidiosa associated with almond leaf scorch disease, 825; population subdivision of Fusarium graminearum from barley and wheat in the upper midwest U.S., 1466; reemerging threat of Phomopsis stem canker sunflower, 990; resurgence of Pseudoperonospora cubensis, 998; spatial and temporal analysis of sudden oak death pathogen in Oregon, 982;

trichothecene genotype composition of Fusarium graminearum populations in New York, 695; virulence and molecular genotype of Puccinia triticina populations in China, 700

Porch, T. G., S1.2, S1.4

Porras, R., S4.106

Porter, D., S4.167

Porter, L. D., S4.113

Porter, P., S4.91

Postharvest pathology and mycotoxins: assessment of aflatoxin and fumonisin contamination of maize in western Kenya, 1250

Postman, J. D., S4.60

Postnikova, O. A., S4.113

Potato late blight. See Phytophthora infestans

Potato psyllid, 189

Potato virus $Y$ : reverse transcription loop-mediated isothermal amplification method for detection, 1270

Potyviridae, 1155

Poudel, R., S4.113

Pouzoulet, J., S4.113

Powdery mildew. See Ampelomyces quisqualis

Powell, C. A., S4.153

Powell, D. H., S4.122

Powell, J. R., S4.131

Pozza, A. A. A., S4.27

Pozza, E. A., S4.27

Pradhan, G. P., 621

Prasad, S., S4.113

Prasanna, B. M., 956

Pratylenchus spp.: predicting disease with soil

DNA and environmental parameters, 1069

Press, C. M., S4.134

Pretorius, Z. A., 872

Price, J. A., 621

Price, P., S2.9
Prieto, K., S4.113

Prithiviraj, B., S4.17

Prodi, A., S4.8

Protacio, C. M., S4.67

Pruitt, H., S2.9

Prunus persica: detection of a new luteovirus in imported trees, 840; fine-scale genetic structure of Monilinia fructicola in tree canopies, 542

Prunus virus T: deep sequencing characterization, 135

Pruss, G. J., S4.162

Pscheidt, J. W., S4.44, S4.66

Pseudomonas spp.

$-P$. cichorii JBC1: light influence on severity in tomato, 412

-P. fluorescens LBUM223: effect on potato yield and common scab symptoms, 1311

Pseudoperonospora cubensis: resurgence of, 998

$\mathrm{Pu}, \mathrm{X} ., \mathrm{S} 4.157$

Puccinia spp.

- P. coronata: QTLs from resistant oat genotypes, 239

-P. graminis f. sp. avenae: resistance gene Rpg5 in barley, 490

-P. graminis f. sp. tritici: emergence and spread of new races, 872; qPCR and fluorescence microscopic analysis of infection process on barley, 707; race TKTTF, phenotypic and genotypic characterization of, 917; race TTKSK, mapping of recessive stem rust resistance gene SrTm4 in wheat, 1347; race TTKSK, resistance in barley, 99; specificity of the 7DL resistance suppressor in Canthatch spring wheat, 477; Sr36- and Sr5-mediated resistance response in wheat associated with guard cell callose deposition, 728; SrND643 resistance gene in wheat, molecular mapping and validation of, 470

- $P$. graminis subsp. graminis: STEMRUST_G model, 35, 45

-P. horiana: systemic infection of chrysanthemum, 91, cover photo: January -P. striiformis $\mathrm{f}$. sp. tritici: characterization of resistance in wheat lines with resistance gene Yr17, 1123; molecular mapping of resistance gene $\operatorname{YrSP}$ in wheat, 1206; race-specific resistance in wheat and characterization of virulence patterns, 1114

-P. triticina: QTL meta-analysis of resistance in wheat, 1585; virulence and molecular genotype of populations in China, 700

Pugliese, M., S4.114

Pumphrey, M., 917

Punja, Z. K., S4.114, S4.119

Pupko, T., S4.40, S4.49

Puppala, N., S4.69, S4.86

Purvis, M., S2.9

Putnam, M. L., S4.30, S4.103, S4.114

$P$ value: future of in plant pathology, 1400

Pyle, J. D., 1146

Pyne, R. M., 885

Pyrenophora teres f. maculata: evaluation of a barley core collection for resistance, 509; seedling resistance in barley, worldwide association mapping, 500

Pyricularia oryzae: effects of silicon on gas exchange and oxidative metabolism in rice leaves infected by, 738; resistance gene $R m g 7$ in tetraploid wheat, 495; wheat resistance gene Rmg8 to Triticum isolates of, 1568

Pythium spp.

- P. irregulare: population structure in Pacific Northwest forest nursery soils, 684

-P. sylvaticum: population structure in Pacific Northwest forest nursery soils, 684

-P. ultimum: population structure in Pacific Northwest forest nursery soils, 684

Qian, G., 588, 1318

Qiao, W., S4.161
Qin, L., 1325

Qiu, D., S4.64

Qiu, W., S4.75, S4.157

Qu, F., 126, S4.65, S4.161

Quan, J., 771

Quantitative real-time polymerase chain reaction (qPCR): analysis of wheat stem infection process on barley, 707; assays for the detection and identification of Meloidogyne enterolobii, 1245; for Fusarium virguliforme in soybean, multilaboratory comparison, 1601

Quantitative trait loci (QTL) meta-analysis: of leaf rust resistance in wheat, 1585

Quesada, T., S4.169

Quesada-Ocampo, L. M., 998, S2.8, S4.23, S4.24, S4.34, S4.96, S4.99, S4.114, S4.125, S4.143

Quezada, T. P., S4.98

Quibod, I., S4.37

Quinone outside inhibitors (QoI) resistance: in

Brazilian populations of Magnaporthe oryzae, 284

Rabiey, M., S4.114

Radmer, L., S4.115

Radwan, O., S4.27

Raffaelea lauricola: effect on leaf gas exchange and xylem sap flow in avocado, 433

Raghavan, C., S4.18

Raghavendra, A. K. H., S4.131

Rahman, A., 748

Rahman, M., S4.115

Raid, R. N., 885, S4.60, S4.109

Raikhy, G., S4.115

Rajakaruna, P., S2.2

Rajasab, A., S4.100

Rajashekara, G., S4.143

Rakotondrafara, A., S4.96, S4.180

Ralstonia solanacearum: race 3 biovar 2 , phylogeographic investigation of and screening for resistance against its core effectors, 597, cover photo: May; type III effectors associated with virulence on tomato and eggplant, 1529

Ramachandran, S., S4.115

Ramadugu, C., S4.71, S4.115

Ramirez, B., S4.33, S4.135

Ramirez, J. H., S2.12

Ramon, M. L., S4.89

Ramos, G. J., S4.116

Ramos, L., S4.116

Ramos Molina, L. M., 1475

Ramsey, J., S4.34, S4.97

Ramularia collo-cygni: mutation in MILDEW

LOCUS $O$ of barley does not affect resistance to grain infection, 1214; review of, 895

Ranabhat, N. B., S4.116

Ranajit Bandyopadhyay, R., S4.8

Randall-Schadel, B., S3.3, S4.126

Rangel, L. I., S4.116

Ranjan, A., S4.116

Rapicavoli, J., S4.116

Rapid isothermal amplification assays: for

detection of Phytophthora spp., 265

Raruang, Y., S4.117

Rascoe, J., S4.22

Rashed, A., 189, 929

Rasmussen, J. B., S4.69

Rath, M., 815, S4.117

Rayapati, N., S4.37

Ray blight of pyrethrum. See Stagonosporopsis tanaceti

Raza, A., S4.7

$R B$ transgene, 1131

Rebollar-Alviter, A., S1.2, S1.3, S4.59

Reddy, K. N., S4.94

Redinbaugh, M. G., 956, S4.174

Redondo, M. A., 1191

Reed, A. J., S4.117

Reed, C., S4.30

Reges, J. T. A., 284

Rehman, A., S4.117 
Reiners, S., S4.178

Reinsel, M. D., S4.11, S4.56

Reisch, B., 1097, 1104

Reitz, S. R., 388

Ren, Z. G., S4.158

REN2 gene, 1104

Rendon-Anaya, M., S4.107

Reniform nematode. See Rotylenchulus reniformis

Resin: effects on spore germination and growth in

Fusarium circinatum, 119

Restrepo, S., S4.25, S4.33, S4.113

Revell, J. M., 91

Reverse-transcription loop-mediated isothermal amplification assay: for Potato virus Y, 1270

Rey, P., S4.138

Reyes-Chin-Wo, S., S4.169

Reyes Gaige, A., S4.117, S4.118

Reyes Navarro, M. A., S4.98

Reyes Salinas, M., S4.98

Reynard, J.-S., 1262

Rezende, J. S., S4.118

$R$ genes: versus nonhost resistance, review article, 580

Rhizoctonia solani: AG-1 IA, emergence in South America via a host shift from rice, 1475; AG-8, predicting disease with soil DNA and environmental parameters, 1069; effects of maple bark biochar on metabolism and damping-off severity, 1334; LAMP assay diagnosis in soybean, 1612

Rhoads, B., S4.91, S4.102

Rhodes, S. C., S2.9, S4.105

Riaño-Pachón, D. M., S4.25

Riaz, K., S4.117, S4.118

Riaz, S., 1104

Richard, D. C., S4.97

Richards, J., S4.47

Richert, B., S4.163

Ridenour, J., S4.156

Riera, N., S4.118

Riggs, J., S4.63

Riley, D., S4.130

Rimbaud, L., 1408

Rios, J. A., S4.23, S4.118

Rios, V. S., S4.118

Ristaino, J., S4.55, S4.118

Ristaino, J. B., 966, S4.27

Ritchie, L., S2.8

Ritenour, M. A., S4.111

Rivard, C., S4.113

Rivera, L. I., S1.4

Rivera, Y., S4.119, S4.122

Rivera Vargas, L. I., S1.1, S1.2, S1.3, S1.4

Rizzo, D. M., S4.19

Rmg7 gene, 495

Rmg8 gene, 1568

Robbins, M. D., 1437

Roberts, D., S4.142

Roberts, D. P., 1325

Roberts, M., S4.129, S4.134

Roberts, P., S4.101

Roberts, P. A., S4.145

Roberts, P. D., 966, S2.5, S2.8

Roberts, R., S4.96

Robertson, A., S4.90, S4.125, S4.173

Robertson, A. E., 1080, S4.81, S4.91, S4.126, S4.140, S4.147, S4.155

Robertson, C. L., S2.2, S2.11, S4.128

Robideau, G., S4.96

Robinson, D., S4.24

Robinson, S. M., S4.119

Robiogovirus, 399

Robson, J., S4.52

Roca-Castillo, L. F., S4.120

Rodoni, B., S4.8, S4.96, S4.131

Rodrigues, F. A., 26, 180, 738, 1050, S4.23, S4.34, S4.118

Rodriguez, G., S4.114, S4.119

Rodriguez, P. A., S4.80
Rodriguez-Campos, E. M., S4.141

Rodriguez-Molina, M., S4.120

Rogers, E. E., 608, S4.10, S4.80

Rojas, A., S4.34, S4.102, S4.120, S4.173

Rollins, J. A., S4.131, S4.157

Rolshausen, P. E., S4.50, S4.113, S4.172

Rolsi, H., S4.59

Romberg, M., S4.119

Romberg, M. K., S3.3, S4.126

Romero, J., S4.120

Romero-Navarro, J. A., S4.33

Ronco, B. L., S4.45

Rong, C. X., S4.144

Rong, L., S4.43

Rooks, S. A., S2.9

Rooney-Latham, S., S4.108, S4.164, S4.165

Roose, M. L., S4.115

Roossinck, M. J., 716

Root-knot nematodes. See Meloidogyne spp.

Roper, C., S4.50, S4.172

Roper, M. C., S4.62, S4.116

Rosa, C., S4.88, S4.161, S4.170

Rosa, J. C. C.-L., 825

Rosenberger, S., S4.120

Rosenberger, S. A., S4.177

Rosenzweig, N., S4.120

Ross-Davis, A., S4.16

Rossi, V., 325, 1090

Rosskopf, E., S4.60

Rosskopf, E. N., S4.26, S4.120

Rosso, L., S4.163

Rotenberg, D., S4.12

Roth, M. G., S4.120

Rothrock, C. S., S2.10, S4.149

Rotich, F., S4.120

Rott, M., S4.112

Rott, P., S4.43, S4.60

Rotylenchulus reniformis: factors affecting spatial distribution in cotton, 674

Roubtsova, T. V., S4.121

Roudet, J., 1090

Roumagnac, P., 716, S4.43

Roundey, E., S4.67

Rouse, D. I., 449, S4.58, S4.179

Rouse, M., 917, S4.65, S4.121

Rouse, M. N., 470, 872, 1347

Rouxel, T., 1288, S4.176

Rowhani, A., 758, 840, S4.5, S4.11, S4.78

Roy, A., 564, 1013, 1277, S1.3, S4.57, S4.121

Royo, C., 1585

RPA190 alleles, 1594

rpg4 gene, 99

Rpg5 gene, 99, 490

Rubagotti, E., S4.97

Rubio, I., S4.5, S4.101, S4.116, S4.121

Ruck, A., S4.111

Rudolph, R., S4.122, S4.147

Ruegger, P., S4.50, S4.154, S4.172

Ruhe, Z., S4.162

Rumsch, V., S4.3

RUN1 gene, 1104

RUN2 gene, 1104

Runde, B., 597

Runge, F., S4.55

Ruongo, S., S4.155

Rupe, J., S4.173

Rupe, J. C., S4.156

Rupp, J. L., S4.121

Rush, C. M., 189, 621, 929

Russell, P., S4.158

Saalau-Rojas, E., S4.112, S4.137

Sabanadzovic, S., S2.10, S4.2, S4.3

Sagawa, C. H. D., 518

Saha, S., S4.145

Sahashi, N., S4.132

Saint-Jean, A., S4.142

Saito, S., S4.121

Sakai, J., 550
Sakalidis, M. L., S4.172

Salacinas, M., S4.105, S4.166

Salas, S. E., S4.122

Salau, A. W., S4.4

Saldarelli, P., 555

Saleem, B., S4.117

Salem, N., S4.103

Salgado, J. D., 295, S4.122

Salgado, S. M., 805

Salgado-Salazar, C., S4.119, S4.122

Salgado-Siclan, M. L., S4.48

Salisbury, J. K., S4.176

Salvatierra, J., 433

Sanatkar, M. R., 947

Sanchez, K. R., S4.122

Sanders, H., S2.3, S4.82

Sandoya, G., 638, 662

Sanogo, S., S4.69, S4.86, S4.122, S4.123

Santa-Barbara, A. E., S4.120

Santiago, A., S4.165

Santiago, T. R., S4.123

Santos, P., 1601

Saparrat, M. C., S4.45, S4.46

Saraiva, R. M., S4.18

Sarra, S., S4.136

Sasaki, K., 525

Sattler, S. E., 786, S4.47

Saude, C., S4.99

Savalle, A., S4.19

Savary, S., S4.177

Savchenko, K. G., S4.123

Saville, A., S4.118

Saville, B. J., 728

Scalco, M. S., S4.27

Scandiani, M. M., S4.123

Schaefer, J., S4.50

Schaerer, S., 1262

Schaffer, B., 433

Schardl, C., S4.13

Scherm, H., 542, S4.19

Schierman, G., S4.124

Schierman, G. T., S4.123

Schilser, D., S4.124

Schlub, R. L., S4.132

Schmale, D. G., S4.167

Schmid, C. J., S4.180

Schmidt, A. M., S4.112

Schnabel, G., 424, S2.9, S4.42, S4.61, S4.63, S4.124

Schneeberger, P. H. H., 1262

Schneider, R, S2.9

Schneider, R. W., 905, S2.2, S2.11, S4.128

Schneider, S. J., S4.85

Schneider, W., S1.3, S3.1, S4.39, S4.124

Schneider, W. L., 564, 1013, 1277, S3.4, S4.41,

S4.43, S4.57, S4.73, S4.121

Schoelz, J., S4.157

Schoelz, J. E., S4.3

Schoen, C., S4.105, S4.166

Schoen, L., 1155

Schoettle, A. W., S4.83

Scholthof, H. B., S4.161

Scholthof, K.-B. G., 1146

Schroeder, B. K., S4.38

Schroeder, F. C., S4.88

Schroeder, K. L., S4.154

Schroeder, N., S4.164

Schubert, T. S., S4.10

Schulte-Geldermann, E., S4.59

Schultz, A., S4.143

Schulze-Lefert, P., S4.173

Schurt, D. A., 1475

Schuster, G., S4.91, S4.124

Schwanck, A. A., S4.177

Schwartz, H., S4.102

Schweigkofler, W., S4.108, S4.165

Sciumbato, G., S4.82

Sclerotinia sclerotiorum: on oilseed rape,

Trichoderma sp. Tri-1 control of, 1325 
Scoglio, C., 947

Scott, I. A. W., 863

Scott, J., S4.125

Scott, J. C., S4.53, S4.124

Scotton, M., 1427

Scruggs, A., S4.23

Scruggs, A. C., S4.125

Scudiero, E., S4.113

Scully, B., S4.154

Scully, B. T., S2.3, S2.13, S4.175

Seal, D. R., S4.156

Searle, C., S4.4

Sechler, A., S3.1, S4.73

Sechler, A. E., S4.43

Secor, G., 966, S4.15, S4.17

See, D. R., 1206

Segal, D., S4.174

Segarra, A. E., S1.1

Seguin, S. G., 35

Sehgal, S. K., S4.2

Seibert, J., S4.141

Seidl, M. F., S4.166

Seidle, A. J., S4.125

Seidl Johnson, A. C., 449

Seifert, K., S4.85

Seijo, T., S4.125

Seijo, T. E., S4.45

Selleck, R., S3.4

Seo, E. Y., S4.83, S4.108, S4.125

Serdani, M., S4.30, S4.103, S4.114

Séré, Y., 1137

Sernett, J., S4.91

Serrano, M., S4.125, S4.140

Serrano-Perez, P., S4.60, S4.120

Serrato-Diaz, L. M., S1.4

Sessa, G., S4.49

Sétamou, M., S4.27, S4.91

Setosphaeria turcica, 1080

Setubal, J. C., S4.157

Severin, A. J., S4.78

Sevgan, S., 956

Sewake, K., S4.129

Sexton, Z., S4.126

Seybold, S. J., S4.121

Sghyer, H., 895

Shaffer, B. T., S4.110, S4.132

Shah, D., S4.70

Shah, D. A., 1400, S4.126

Shahrtash, M., S2.9

Shakya, S. K., 230, S4.141

Shan, W., 771

Shang, Q., S4.147

Shang, Q. X., S4.158

Shang, W.-J., 220

Shao, D., S4.150

Shao, J., 564, 1013, 1277, S1.3, S4.33, S4.57, S4.113, S4.121

Sharifi-Tehrani, A., S4.90

Sharka: assessing the mismatch between

incubation and latent periods, 1408

Sharma, A. K., 1043

Sharma, R., S4.169

Sharma, S., S4.156, S4.165

Sharma Poudel, R., S4.126

Sharma Poudyal, D., S4.126

Sharpshooters: direct evidence for egestion and salivation of Xylella fastidiosa, 608

Shatter, R. G., S4.153

Shatters, R., 608, S4.38

Shaw, D. C., S4.169

Shaw, M. W., S4.41, S4.114

Shekels, T. J., S4.133

Shelley, B., S3.5

Shen, D., S4.134

Shen, H., S4.157

Shen, Q., S4.175

Shen, W., S4.84

Sheng, H., S4.126

Shennan, C., S4.59, S4.120
Shepherd, C. P., S4.122

Sherman, D., S4.124

Sherman, D. J., S3.4

Sherrier, J., S3.2

Shew, B., S3.3, S4.85

Shew, B. B., S4.126

Shew, H. D., S2.6, S4.74, S4.91

Shi, J., S4.107

Shi, S., S4.76, S4.127

Shiel, P., S4.130

Shigyo, M., 525

Shih, H. T., S4.133

Shim, H., S4.99

Shim, K., S4.112

Shimwela, M. M., S4.127

Shinohara, H., S4.70

Shirako, Y., S4.82

Shirley, B., S4.19

Shishkoff, N., S4.127

Shiu, L. Y., S4.28

Shivas, R. G., S4.137

Short, D. P. G., 80, 638

Shrestha, B. K., S4.127

Shrestha, U., S4.127

Shuey, L., S4.132

Shugart, H. J., 608

Siddiqui, N., S4.135

Siddrame, G., S4.38

Sierotzki, H., 998

Signficance level ( $P$ value): future of in plant pathology, 1400

Sikdar, P., S4.127

Silberstein, T. B., 35

Silicon: effects on gas exchange and oxidative metabolism in rice leaves infected by Pyricularia oryzae, 738; induced systemic defense responses in perennial ryegrass against Magnaporthe oryzae infection, 748

Silué, D., S4.120

Silva, C. N., 246

Silva, D. D., S4.130

Silva, E. C., S4.128

Silva, G. M., S4.128

Silva, L. C., S4.23

Silva, M. C. M., 628

Silva-Rojas, H. V., S1.2, S1.3, S4.59, S4.128

Silver nanoparticles: synthesized with aqueous extract of Artemisia absinthium, inhibition of Phytophthora parasitica and P. capsici by, 1183

Sim, J., S4.116

Sim, S.-C., 1437

Simbaña, L. L., S1.4

Simko, I., 1220

Simmon, I., S4.169

Simmons, C., 1362

Simon, A. C., S4.128

Simon, A. C. M., S4.85

Simon, J. E., 885

Simons, K., S4.160

Sims, A. L., S4.26

Singh, D., 490

Singh, M., S4.101

Singh, P., S4.128

Singh, R. P., 470, 872, 917, S4.68

Singh, S., 470

Sinn, J., S4.93

Siozios, S., 199

Sipes, B., S4.129

Siri, M. I., S4.31, S4.78

Siritunga, D., S4.156

Sisco, R., S4.42

Sit, T. L., S4.53

Skaltsas, D., S4.129

Skantar, A., S4.113

Sklarczyk, D., S4.164

Slanec, T., S4.10

Slininger, P., S4.124

Slinski, S. L., 119

Slupsky, C., S4.97
Small, I. M., 342, 1545, 1594, S4.129

Small interfering RNAs (siRNAs): targeting unc-15 in sweet potato confers resistance to Ditylenchus destructor, 1458

Smart, C. D., 169, 966, 1355, S1.5, S4.24, S4.51, S4.76, S4.135, S4.177, S4.178

Smart, T., S4.49, S4.58, S4.129, S4.134

Smith, A. L., S4.86

Smith, C., S4.123

Smith, D., S4.16

Smith, D. L., S4.116, S4.148

Smith, D. R., S1.6

Smith, G., S4.158

Smith, G. R., 863

Smith, H., S4.84

Smith, J., S4.129, S4.156

Smith, J. A., S4.38, S4.61, S4.107, S4.169

Smith, K. M., S3.1

Smith, K. P., 99

Smith, O. P., S3.3, S3.5

Smith, R., S4.8

Smith, S., S2.3, S4.39

Smith, S. M., S2.10

Smolinksi, T., S4.112

Sniezko, R. A., S4.83

Snover-Clift, K., S4.130

Soares, L. F., S4.18

Soares, L. S., S4.51

Soby, S., S4.57, S4.137

Soby, S. D., S4.39

Sogatella furcifera: quantitative analysis of Southern rice black-streaked virus in and threshold for transmission, 550

Soil DNA: predicting cereal root disease with, 1069

Solanki, S., S4.126

Solanum spp.: testing taxonomic predictivity of foliar and tuber resistance to Phytophthora infestans in wild relatives, 1198

-S. lycopersicon: association analysis for bacterial spot resistance in, 1437; light influence on disease severity of Pseudomonas cichorii JBC1, 412; reverse transcription loop-mediated isothermal amplification method for detection of Potato virus Y, 1270; type III effectors associated with Ralstonia solanacearum virulence, 1529

$-S$. melongena: cell death response to Ralstonia solanacearum race 3 biovar 2, cover photo: May; type III effectors associated with Ralstonia solanacearum virulence, 1529

-S. tuberosum: age-dependent correlation of tuber blight resistance with $R B$ transgene transcript levels, 1131; effect of Pseudomonas fluorescens LBUM223 on yield and common scab symptoms, 1311; effects of potato-pysllidvectored 'Candidatus Liberibacter solanacearum' infection on leaf and stem physiology, 189; evaluation of BlightPro decision support system for management of blight, 1545; population structure of Phytophthora infestans in a germplasm nursery, 771; tolerance to 'Candidatus Liberibacter solanacearum' in germplasm, 1573

Son, M., S4.155

Song, B., 1612

Song, F., S4.175

Song, J. H., S4.130

Song, Q., S4.108

Song, T., S4.130

Song, Z., 588

Sood, S., S4.60

Sorghum bicolor: effect of waxy gene on fungal infection of grain, 786; identification of a QTL for plant height in, 1522; inheritance and identification of a major QTL for Meloidogyne incognita resistance in, 1522

Soriano, J. M., 1585

Soriano, M. C., S4.164 
Soubeyrand, S., 1408

Sousa, L. L., S4.50

Southern rice black-streaked virus: in Sogatella furcifera, quantitative analysis and threshold for transmission, 550

Souza, A. G. C., S4.130

Souza, H. S., S4.51

Souza, M. A., S4.118

Souza, R. M., S4.128

Souza-Junior, J. D. A., 628

Sowiński, M., 1270

Soybean rust. See Phakopsora pachyrhizi

Soybean sudden death syndrome. See Fusarium virguliforme

Spadaro, D., S4.53

Spinacia oleracea: frequency of Verticillium species and subsequent transmission to lettuce crops, 80

Spolti, P., 246, 695

Spooner, D. M., 1198

Spot form net blotch. See Pyrenophora teres f. maculata

Spraker, J., S4.163

Spring, O., S4.55, S4.169

Spurlock, T. N., S2.10, S4.149

Sreenivasan, T. N., S4.20

Sridharan, M., S4.167

Srinivas, G., S4.173

Srinivasan, R., S4.130

SrND643 gene: in wheat, molecular mapping and validation of, 470

Srour, A. Y., 1601, S4.131

SrTm4 gene: mapping in wheat, 1347

Ssemakula, G. N., S4.179

Stachecka, J., 1270

Stack, J. P., S4.8, S4.22, S4.96, S4.97, S4.117, S4.118, S4.131

Stafford-Banks, C. A., S4.105

Stagonospora nodorum blotch: effects of wheat residue on severity and yield in winter wheat, 1417

Stagonosporopsis spp.: genetic diversity and population structure, 815

-S. tanaceti: changes in genetic composition in Australian pyrethrum, 358

Standish, J. R., S2.10, S4.2, S4.20

Stanković, I. M., S4.21, S4.76

Stanosz, G. R., S1.6

Stavornvisit, P., S4.131

Stead, D., S4.5

Steadman, J. R., S4.41, S4.131

Steere, L., S4.120

Stefanelli, S., S4.8

Steffenson, B. J., 99

Steinke, K., S4.120

Steinrucken, T. V., S4.131

Stelly, D. M., S4.145

Stem nematode. See Ditylenchus destructor

STEMRUST_G model, 35, 45

Stem rust resistance genes: mapping and validation $S r N D 643$ in wheat, 470; mapping of SrTm4 in wheat, 1347; Sr36- and Sr5-mediated resistance response associated with guard cell callose deposition in wheat, 728

Stenger, D. C., S4.21

Stenocarpella macrospora: effects on gas exchange and chlorophyll $a$ fluorescence in maize, 26

Stensvand, A., S4.9

Stephenson, R. C., S4.3

Sternberg, P. W., S4.88

Stevenson, K., S2.8

Stevenson, K. L., S2.3, S4.17, S4.82

Stewart, C., S1.5

Stewart, C. L., 1146

Stewart, J., S4.35, S4.132

Stewart, L. R., 833, 956, S4.65

Stewart, S. D., S2.2

Still, W., S3.1
Stobbe, A., S4.39

Stockwell, V., S4.73, S4.132

Stoepler, T., S4.80

Stoghill, P., S4.44

Stojšin, V., S4.21

Stone, A., S4.124

Stone, A. L., 564, S3.4

Stover, E., S4.38, S4.111, S4.115

Strausbaugh, C. A., S4.132

Strauss, S. L., 460

Strayer, A. L., S4.132

Strelkov, S. E., 764, S4.26, S4.51, S4.62

Streptomyces spp.: Pseudomonas fluorescens

LBUM223 reduces common scab symptoms in potato, 1311

Streptomycin resistance: characterization in

Erwinia amylovora isolates from California, 1302

Stubblefield, S., S4.80

Studholme, D. J., 597

Stukenbrock, E. H., S4.172

Stulberg, M. J., S4.132

Stump, W. L., S4.133

Sturrock, R., S4.83

Su, C. C., S4.133

Suarez, S. N., S4.133

Subbarao, C. S., S4.133

Subbarao, K. V., 80, 638, 662, 852, S4.77, S4.133, S4.176

Subbotin, S. A., S4.133

Subedi, A., S4.134

Sudarshana, M. R., 840, 1026, S4.11, S4.65,

S4.78, S4.134

Sudden oak death. See Phytophthora ramorum

Suffert, F., S4.142

Sugino, K., S4.180

Sullivan, K. L., 863

Sullivan, M., S4.129, S4.134

Sullivan, M. J., S4.15

Sultana, V., S4.10, S4.40, S4.102

Sumabat, L., S4.134

Sun, J., 771

Sun, L., S4.81

Sun, W., S4.175

Sunadaraj, S., S4.130

Sundin, G. W., 852, 1302, S4.25

Surujdeo-Maharaj, S., S4.20

Susaimuthu, J., 399

Susanne, V., S4.89

Suslow, K., S4.108, S4.165

Suzuki, N., S4.157

Svanella-Dumas, L., 1155

Sweany, R. R., S2.10, S4.110, S4.134

Sweeney, C. R., S4.58

Sweeney, R., S4.49

Swiecki, T. J., S4.164, S4.165

Szabo, L., S4.65

Szabo, L. J., 872, 917

Szurek, B., S4.37

T4P-dependent twitiching motility: PilG regulation in Lysobacter enzymogenes, 1318

Tabe, L., 477

Tabima, J. F., S4.134, S4.172

Tadesse, K., 917

Tagle, A. G., 495, 1568

Tahir, M. N., S4.135

Tahtamouni, E., S4.122

Takeda, F., S4.64

Takumi, S., 1568

Talajoor, M., 477

Talbot, N., S4.120

Tally, A., S2.6, S2.11, S4.4, S4.56, S4.76, S4.135,

S4.136, S4.148

Tamang, P., 500, 509

Tamborindeguy, C., S4.81

Tan, S. H., S4.135

Tan, W., S4.132
Tan, Y. P., S4.137

Tanacetum cinerariifolium: changes in genetic composition of Stagonosporopsis tanaceti population, 358

Tanaka, S., 525

Tancos, M. A., 169, S4.135

Tandem repeat variability: genetic diversity in

'Candidatus Liberibacter asiaticus', 1043

Tani, A., S4.157

Tantiwanich, Y., 388

Tao, J., S4.20

Taški-Ajduković, K., S4.21

Tatagiba, S. D., 180

Tate, D., S4.135

Tatineni, S., 1496, S4.54, S4.135, S4.150

Taylor, C. G., S4.46, S4.94, S4.144

Taylor, G. H., S4.171

Taylor, J., S4.19

Taylor, N. J., S4.109

Taylor, O. J., S4.64

Taylor, P. W. J., 358

Techniques: diagnosis of soybean seedling blight and soybean charcoal rot using LAMP assays, 1612; DNA macroarray for detection and identification of fungal pathogens causing decline of young grapevines, 1373; improved diagnosis and quantification of Fusarium virguliforme, 378; multilaboratory comparison of qPCR assays for Fusarium virguliforme in soybean, 1601; qPCR and fluorescence microscopic analysis of wheat stem rust infection process on barley, 707; rapid isothermal amplification assays for detection of

Phytophthora spp., 265

Tedford, C. E., S4.136

Tekete, C., S4.37, S4.136

Tekik, A. G., S4.22

Tellier, A., 895

Tenuta, A. U., S4.69, S4.173

Teodorović, S. D., S4.21, S4.76

Teshler, I., S4.92

Tessmann, D. J., 246

Tesso, T. T., S4.11, S4.12

Testen, A. L., S4.136

Textor, K., 1214

Thannhauser, T., S4.97

Tharreau, D., S4.120

Thébaud, G., 1408

Theil, S., 1155

Thekke-Veetil, T., S4.2

Themis, M. J., S4.121

Thies, J. A., S4.136

Thiessen, L., S4.136

Thines, M., S4.77

Thomas, A., S4.137

Thomas, W. J., S4.30, S4.103

Thomas-Sharma, S., S4.137

Thomma, B., S4.17

Thompson, J., S4.137

Thompson, S. M., 863, S4.137

Thomson, J., S4.137

Tian, F., S4.82

Tian, M., S4.150

Tian, P., S2.10

Tian, T., S4.13, S4.111

Tian, Y., 771

Tiedje, J., S4.172

Tiedt, L. R., S4.133

Timilsina, S., S2.10

Tirajoh, A., S4.114

Tobacco etch virus, 18

Todd, J., 833

Todd, T., S4.32

Togawa, R. C., 628

Tohamy, A. M., S4.138

Tolbert, A. C., S2.10

Tolin, S. A., S4.173

Tomaso-Peterson, M., S2.10, S2.11, S2.12, S4.2, S4.20, S4.148, S4.181 
Tomato chlorotic spot virus: emergence in southeastern U.S., 388

Tomlinson, A. J., 110

Tonnessen, B. W., S4.138

Tononi, P., 199

Toomajian, C., S4.118, S4.155

Torrance, T. N., S2.11

Torres, R., S4.37

Tosa, Y., 495, 1568

Tosi, L., 199

Toth, I., S4.105

Tournas, V. H., S4.138

Toussaint, V., S4.4, S4.35

Towata, T., 550

Tran, T. M., S4.138

Tran, T. T., S4.107

Transcriptional programming: of Ampelomyces quisqualis during host-induced germination 199

Trapero-Casas, A., S4.97, S4.106, S4.120, S4.141

Travadon, R., 255, S4.78, S4.138

Travers, P., S4.54, S4.175

Trebicki, P., S4.32, S4.139

Tredway, L., S4.23

Trent, M., 316

Tri, A., S4.91

Trichoderma sp. Tri-1: control of Sclerotinia sclerotiorum on oilseed rape, 1325

Trichothecene genotype composition: of Fusarium graminearum populations in New York, 695

Trick, H. N., S4.121

Trigiano, R., S4.55

Trippe, A., S4.165

Triticosecale: genetic architecture of Fusarium head blight resistance, 334

Triticum aestivum: biological activity of in vitro transcripts of wild-type and fluorescent protein-tagged Triticum mosaic virus in, 1496; effects of Fusarium head blight on grain yield and test weight, 295; effects of post-anthesis moisture patterns on Fusarium head blight development and DON accumulation, 210; effects of wheat residue on severity of Stagonospora nodorum blotch and winter wheat yield, 1417; physiological responses of hard red winter wheat to Wheat streak mosaic virus infection, 621; population subdivision of Fusarium graminearum in the upper midwest U.S., 1466; Pyricularia oryzae resistance gene Rmg7 in tetraploid wheat, 495; Pyricularia oryzae resistance gene $R m g 8$ in hexaploid wheat, 1568; QTL meta-analysis of leaf rust resistance in, 1585; specificity of the 7DL rust resistance suppressor in Canthatch spring wheat, 477; Sr36- and Sr5-mediated resistance response to Puccinia graminis f. sp. tritici associated with guard cell callose deposition, 728; stem rust

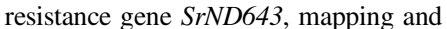
validation of, 470; stem rust resistance gene SrTm4, mapping of, 1347; stripe rust resistance, characterization in lines with resistance gene $\operatorname{Yr17}$, 1123; stripe rust resistance, race-specific, 1114 ; stripe rust resistance gene $Y r S P$, mapping of, 1206

Triticum mosaic virus: biological activity of in vitro transcripts of wild-type and fluorescent protein-tagged virus in wheat, 1496

Trivedi, P., S4.81

Tron, F., S4.129

Truchon, A., S4.139

Truco, M.-J., 1220

Truke, M. J., S1.3, S1.5

Truke Arango, M. J., S1.6

Tsai, W. S., S4.139

Tsai, Y.-L., 160

Tsay, J. S., S4.151

Tsuchida, C., 1220, S4.77, S4.169

Tubbs, R. S., S2.12

Tubbs, T., S4.139
Tuberosa, R., S4.8

Tung, C.-Y., 160

Tungadi, T., S4.3

Turechek, W. W., 388, S4.50, S4.75, S4.145

Turina, M., S4.88

Turini, T., S4.139

Turnbull, G. D., S4.26, S4.62

Turner, S., S4.39

Tweddell, R., S4.4, S4.35

Twizeyimana, M., S4.91, S4.180

Twomey, M., S4.49

Tychon, B., S4.40

Tyler, B. M., S4.134

Tyler, D. D., S4.94

Tylka, G. L., S4.14, S4.22, S4.140

Type III effectors: associated with Ralstonia

solanacearum virulence on tomato and eggplant, 1529

Type IV secretion system, 160

Tzanetakis, I. E., S2.5, S4.2, S4.36, S4.60

Tzeng, J. Y., S4.35, S4.151

Uchanski, M., S4.122

Uchida, J., S4.38, S4.123, S4.124, S4.140

Uddin, W., 748

Ulibarri, C. L., S4.6

Ullman, D. E., S4.28, S4.105

Ulloa, M., S4.145

Umaharan, P., S4.20

unc-15 gene, 1458

Uncinula necator. See Erysiphe necator

Uppala, S. S., S4.140, S4.159

Upper, D., 45

Urbez-Torres, J. R., 1373, S4.112, S4.140

Urbina-Cortes, T. B., S4.59

Uribe-Cortes, T. B., S4.128

Urochloa: emergence of Rhizoctonia solani AG-1

IA in South America via a host shift from rice, 1475

Uyemoto, J. K., 840, S4.11, S4.78, S4.134

Uzest, M., S4.170

Vaccinium spp.: Xylella fastidiosa isolates from subsp. multiplex and fastidiosa cause disease under greenhouses conditions, 855

- V. corymbosum: characterization of Alternaria spp. associated with fruit rot in California, 1555

- V. sect. Cyanococcus: population structure of

Monilinia vaccinii-corymbosi in the U.S., 533

Vaghefi, N., 358

Vahling-Armstrong, C., S4.38

Vaillancourt, L. J., S2.7

Valdés, I., S4.25

Valdes, S., S4.157

Vale, F. X. R., 738

Valenicia-Torres, N., S4.95

Valent, B., S4.32, S4.111

Valentini, G., S4.108

Valero, G., S4.46

Vallad, G., S2.6, S4.70, S4.105

Vallad, G. E., 638, S2.6, S2.8, S2.10, S4.141

Vallance, J., S4.138

van Bruggen, A. H. C., 230, S4.100, S4.127, S4.141

Vance, V., S4.162

van den Berg, E., S4.133

van den Berg, F., S4.73

van den Bosch, F., S4.73

Van den Langenberg, K. M., 998

Vandermeer, J., 1164

Vander Negri, Pola, D., S4.29

Vanderpool, A., S4.55

van der Wolf, J. M., S4.80

van Eck, L., S4.141

van Hest, P, 1573

van Kan, J. A., S4.41

van Klinken, R. D., S4.131

van Pelt, A., S4.147

Van Zyl, G., S4.71
Varady, E., S4.135, S4.141

Varela, I., S1.3

Vargas, A., S1.4

Vargas, A. I., 433

Vargas, W., S4.123

Varnedore, T., S4.19

Varo-Suarez, A., S4.141

Varshney, R. K., S2.3, S2.11, S4.169, S4.175

Vásquez, J. J., S4.113

Vasquez-Siller, L. M., S4.141

Vazquez, O., S4.85, S4.142

Vector-borne diseases: assessing the mismatch

between incubation and latent periods, 1408

Vega, B., S4.71, S4.101

Vega, B. P., S2.3

Veltri, D., S4.88, S4.119

Vera Bahima, J., S4.45

Vera Cruz, C. M., S4.18, S4.37

Verchot, J., S2.2

Verchot-Lubicz, J., S2.12

Verdier, E., S4.78

Verdier, V., S4.18, S4.37, S4.136, S4.149

Verma, R., S4.142

Vernon, M., S4.97

Verticillium spp.: in spinach fields and transmission to subsequent lettuce crops, 80

$-V$. dahliae: detection with wet-sieving qPCR, 220; factors affecting dynamics of

microsclerotia in field soils, 658; transmission from infested spinach to subsequent lettuce crops, 80

$-V$. longisporum: factors affecting dynamics of microsclerotia in field soils, 658; lineage differences in virulence and pathogenecity, 662 Vescio, K., S4.54

Viana, A. A. B., 628

Victoria, J. I., S4.8, S4.23

Vida, G., 797

Vidal, T., S4.142

Vidalakis, G., S4.33, S4.50, S4.107, S4.135, S4.141

Vieira, J. R., Jr., 1475

Vieira, P., S4.142, S4.181

Vieira Gody, C., S4.33

Viljoen, A., S4.166

Villalobos-Escobedo, J. J., S4.107

Villamor, D. E. V., 399

Villari, C., S4.142

Vinatzer, B. A., 597, S4.93, S4.143

Vines, P., S4.2

Vines, P. L., S2.11, S4.181

Virology: alignment-guided mutagenesis of an attenuated, cross-protective Pepino mosaic virus variant, 126; Bean common mosaic virus isolate able to overcome the $b c-3$ resistance allele in common bean, 1487; biological, serological, and molecular characterization of Grapevine leafroll-associated virus 4, 1262; biological activity of in vitro transcripts of wild-type and fluorescent protein-tagged Triticum mosaic virus in wheat, 1496; characterization of Jatropha mosaic virus from the Dominican Republic, 141; complete nucleotide sequence of Artichoke latent virus places it the genus Macluravirus, 1155; complex relationships between viruses, hosts, and vectors related to citrus leprosis, 1013; Cucurbit aphid-borne yellows virus resistance in melon ascension TGR-1551, 1389; deep sequencing characterization of Prunus virus T, 135; detection of a new luteovirus in imported nectarine trees, 840; emerging threat of grapevine red blotch-associated virus, 1026, cover photo: July; genetic variability of Grapevine Pinot gris virus and association with grapevine leaf mottling and deformation, 555; Groundnut ringspot virus and Tomato chlorotic spot virus emergence in southeastern U.S., 388, cover photo: March; history and diversity of Citrus leprosis virus recorded in herbarium 
specimens, 1277; identification and molecular characterization of nuclear Citrus leprosis virus, 564; infectious Maize rayado fino virus from cloned cDNA, 833; metagenomics of plant viruses, review article, 716; next-generation sequencing versus biological indexing for detection of grapevine viral pathogens, 758; Panicum mosaic virus and its satellite virus infection of switchgrass breeding plots, 1146, cover photo: August; possible new genus within Betaflexiviridae, 399; quantitative analysis of Southern rice black-streaked virus in Sogatella furcifera, 550; reverse transcription loop-mediated isothermal amplification method for detection of Potato virus $Y, 1270$

Vitis spp.

-V. rupestris B38: quantitative resistance to Erysiphe necator, 1097

-V. vinifera: biological, serological, and molecular characterization of Grapevine leafroll-associated virus 4, 1262; DNA macroarray for detection and identification of fungal pathogens causing young vine decline, 1373; emerging threat of grapevine red blotch-associated virus, 1026 , cover photo: July; environmental conditions affect Botrytis cinerea infection of berries more than strain or transposon genotype, 1090; factors affecting Botrytis cinerea infection on inflorescences and young berry clusters, 325; genetic variability of Grapevine Pinot gris virus and association with leaf mottling and deformation, 555; next-generation sequencing versus biological indexing for detection of viral pathogens, 758

Voeltz, M., S4.135

Vogel, K. P., 1146

Volke-Haller, V., S1.2

von Reuss, S. H., S4.88

von Tiedemann, A., 662, 1137

Vorholt, J., S4.173

Vovlas, N., S4.133

Vrisman, C. M., S4.143

Vu, S., S4.143

Vućurović, A. B., S4.21, S4.76

Vy, T. T. P., 1568

Waalwijk, C., S4.166

Wade, L., S4.55

Wadl, P., S4.55

Walcott, R., S4.40, S4.65

Walcott, R. R., S4.128

Walenta, D., S4.70

Walenta, D. L., S4.124

Walker, D., S2.5

Walker, D. R., 905, S4.61

Walker, L., S4.143

Walker, M. A., 1104

Walker, N. R., 656

Walker, S., S4.116, S4.122

Wallace, E. C., S4.143

Waller, T. J., S1.8

Wallhead, M., S1.6, S4.167

Wallis, C., S4.47, S4.143

Wallis, C. M., 189, 748, 1573

Walsh, E., S4.144

Walters, T., S4.147

Wamishe, Y., S4.145

Wan, A., 477, S4.69, S4.144, S4.151

Wan, A. M., 1206

Wang, B. T., S4.61

Wang, C., S4.145, S4.147

Wang, C. J., S4.30

Wang, D. H., S4.91, S4.180

Wang, G. L., S4.95, S4.120

Wang, H., 1437, 1522, S2.11, S4.145

Wang, J., 378, 1601, S4.120, S4.144, S4.145, S4.146, S4.155

Wang, J. S., S4.163
Wang, L., S4.77, S4.145

Wang, M., S4.69, S4.144, S4.151, S4.175

Wang, M. N., 1206

Wang, N., S4.38, S4.56, S4.61, S4.65, S4.81,

S4.83, S4.113, S4.118, S4.157, S4.159

Wang, N. Y., S4.145, S4.157

Wang, P., S4.62

Wang, Q., S4.156

Wang, R., 482

Wang, S., S4.18

Wang, X., 728, S4.88, S4.145, S4.181

Wang, Y., 1612, S4.144

Wangai, A., 956

Wanjala, B., 956

Wanner, L. A., S4.146

Wantoch, S., S4.142

Wanyera, R., S4.65

Ward, B. M., S2.2, S2.11, S4.128

Ward, T. J., 246, 1466

Ward-Gauther, N., S4.146

Warghane, A., 1043

Warner, A. J., S4.146

Warren, J., S4.170

Washburn, B. K., S4.10

Watanabe, S., S4.27

Watkinson, S., S4.147

Watson-Selph, A. K., S2.9

Watters, K., S4.137

waxy gene, 786

Wayadande, A., S4.124

Webb, K. M., S4.146

Webb, M. A., S2.12

Webster, B. J., S4.146

Webster, C. G., 388

Webster, T. M., S2.11

Weerasooriya, D. K., S4.11

Wegulo, S. N., S4.150

Wehner, T. C., 998

Wei, F., 220

Wei, G., 564, S4.144, S4.146

Wei, H., S4.84

Wei, J., 1362

Wei, Q., S4.117

Wei, W., S1.2

Wei, Y., S4.147

Wei, Y. M., S4.158

Wei, Z., 1458

Weieneth, L. K., S4.147

Weiland, J., S4.76

Weiland, J. E., 684, S4.147

Weiland, J. J., 833

Weisberg, A., 597

Weisberg, A. J., S4.143

Weisz, R., 1417

Welbaum, G., S4.40

Weldon, W., S4.179

Weller, D. M., S4.147

Welliver, R., S4.166

Wells, C., S4.81

Wells, L., S4.112, S4.137

Wen, A, 863

Wen, L., S4.147

Wenbo, M., S4.107

Wen-Hsin, C., S4.62

Wester, D., S4.91

Westerdahl, B., S4.147

Wet-sieving qPCR: detection of Verticillium dahliae, 220

Wharton, P., S4.37

Wharton, P. S., S4.148, S4.149

Wheat blast. See Magnaporthe oryzae

Wheat powedry mildew. See Blumeria graminis

f. sp. tritici

Wheat stem rust. See Puccinia graminis

f. sp. tritici

Wheat streak mosaic virus: physiological

responses of hard red winter wheat to infection, 621

Wheeler, T. A., S4.150
White, J. C., S1.7

Whitebacked planthopper. See Sogatella furcifera Whitfield, A., S4.12

Whitfield, A. E., S4.89, S4.170

Whitten Buxton, K., S4.148

Wick, R., 885

Wick, R. L., S1.6

Wicker, E., 597, 1529

Wickert, K., S4.148

Wideman, R., 433

Widholm, J. M., S4.109

Widmer, T. L., S3.5

Wieman, M. S., S4.114

Wiglesworth, M. D., S2.6, S2.11

Wijayasekara, D. S., S2.12

Wijeratne, S., 1437

Wilcox, W. F., 370, S4.56

Wildung, M. R., S4.49

Wilkerson, T., S2.12, S4.148

Wilkerson, T. H., S4.88

Willbur, J. F., S4.148

Williams, B. W., S2.12

Williams, P., S4.58

Williams, W. P., S4.149

Williamson, V. M., 350, S4.88

Willis, R., S4.141

Willmann, R., S4.149

Willocquet, L., S4.177

Wilson, C., S4.125

Wilson, K. D., S4.149

Wilson, R. A., S4.89

Win, J., S4.150

Windels, C. E., S4.26

Windham, G. L., S4.149

Wintermantel, W. M., S4.13, S4.70

Wiriyajitsomboon, P., S4.149

Wise, K. A., S4.69, S4.90, S4.173

Wisser, R. J., S4.95

Withers, S., S4.24, S4.114

Wof, T., S4.80

Woldeab, G., 917, S4.65

Wolfenbarger, S., S4.49

Wolford, S., S4.64

Woloshuk, C., S4.102, S4.139

Wong, J., S4.169

Wong, L., S4.38

Wonni, I., S4.149

Wood, B. W., S4.17

Wood, K., S4.169

Woodell, L., S4.124

Woodhall, J., S4.148, S4.149

Woods, J., S4.49

Woodward, J., S2.12

Woodward, J. E., S4.150

Wordell, Filho, J. A., 26

Workneh, F., 189, 929

Wosula, E. N., S4.150

Woudt, B., S4.149

Wright, A., S4.116

Wright, D. L., 905

Wroblewski, T., 597

Wu, B., S4.38

Wu, B.-M., 638

Wu, D., S4.150

Wu, G., 588, 1362, S4.150

Wu, J., S4.150

Wu, J. J., S4.35, S4.151

Wu, Y., S4.33, S4.129

Wu, Z. B., S4.151

Wuest, C. E., S4.151

Wuriyangham, H., S4.170

Wyatt, M., S4.27

Wydra, K., 1137, S4.37

Wyenandt, C. A., 885

Wyka, S., S1.8

Wyka, S. A., S4.177

Xanthomonas spp.: association analysis for bacterial spot resistance in tomato, 1437 
-X. campestris pv. vitians: effects of host genotype and hypersensitive reaction infection in lettuce, 316

-X. oryzae pv. oryzidola Rs105: novel

DSF-controlled virulence-associated genes, 588

Xi, P., S4.81

Xia, C., S4.151

Xiang, B., S4.32

Xiang, M., S4.152

Xiang, N., S2.12, S4.151

Xiang, Y., S4.151

Xiao, C. L., 1555, S4.4, S4.121, S4.168

Xiao, C.-L., 638

Xie, L., 1325

Xie, Q, S4.175

Xie, X., S4.81

Xing, Y., S4.59, S4.137

Xiong, Z., S4.32, S4.76, S4.127, S4.155

$\mathrm{Xu}, \mathrm{H} ., \mathrm{S} 4.152$

$\mathrm{Xu}, \mathrm{J} ., \mathrm{S} 2.13$

$\mathrm{Xu}, \mathrm{L} ., \mathrm{S} 4.152$

$\mathrm{Xu}, \mathrm{Q}, \mathrm{S} 4.152$

Xu, S. S., S4.69

$\mathrm{Xu}, \mathrm{X} ., 1174$

$\mathrm{Xu}, \mathrm{X} . \mathrm{M} ., \mathrm{S} 4.61$

Xu, X.-M., 220

$\mathrm{Xu}, \mathrm{Y} ., \mathrm{S} 4.152$

$\mathrm{Xu}, \mathrm{Z} ., \mathrm{S} 4.81$

Xue, D., S4.82

Xue, Q., 621

Xylella spp.

-X. fastidiosa: associated with almond leaf scorch disease, population structure, 825 ; direct evidence for sharpshooter egestion and salivation by sharpshooters, 608

-X. fastidiosa subsp. fastidiosa: infection of southern highbush blueberry under greenhouse conditions, 855

-X. fastidiosa subsp. multiplex: infection of southern highbush blueberry under greenhouse conditions, 855

Xylem sap flow: in avocado, effect of Raffaelea lauricola on, 433

Yaghmour, M. A., S4.121

Yan, G. P., S4.153

Yan, Q., S4.110, S4.159

Yan, Y., S4.67

Yanev, G., S4.142

Yánez-Mendizábal, V., S4.153

Yang, C., S4.153

Yang, C. H., S4.82

Yang, J. I., S4.172

Yang, J.-I., S4.154

Yang, J.-R., 220

Yang, L., S2.3, S2.13, S4.154, S4.175

Yang, S. L., S4.153
Yang, W., 1437

Yang, X., S4.30, S4.100, S4.154, S4.175

Yasin, M., S4.117

Yates, D. I., S4.75

Yates, J., S4.164

Ye, W., S4.153

Yeh, C. C., S4.154

Yen, A., S4.139

Yencho, C. G., S4.179

Yendrek, C., S4.27

Yermiyahu, U., 1059

Yin, C., S4.154

Yin, J., 771

Yin, X., S4.94

Yokomi, R. K., S4.27, S4.154

Yokota, K., S4.46, S4.70

Yoon, J. Y., S4.29, S4.155

Yoon, M. J., S4.99

Yordem, B., S4.163

Yoshida, K., 550

Young, J. C., S4.20

Young-Kelly, H., S2.2, S4.22

Young vine decline: DNA macroarray for detection and identification of fungal pathogens causing, 1373

Yrl7 gene, 1123

YrSp gene: molecular mapping, 1206

Yu, C., 1325

Yu, F., S4.130

Yu, J., S4.67, S4.87, S4.155

$\mathrm{Yu}, \mathrm{N} ., \mathrm{S} 4.155$

Yu, S.-H., S4.125

Yuan, X., S4.82

Yue, W., S4.118, S4.155

Yue, X., S4.47

Yuen, G. Y., 1146, 1318

Yusuf, S., S4.155

Zaccaron, A., S4.156

Zaccaron, M. L., S4.156

Zahariev, M., S4.85

Zakaria, L., S4.156

Zakharov, F., 119

Zakri, A. M., S4.7

Zamany, A., S4.83

Zambrana-Echevarria, C., S4.156

Zamora-Díaz, M. R., S4.141

Zanutto, C. A., S4.51

Zapiola, J., S4.123

Zasada, I. A., S4.76, S4.134, S4.147

Zea mays: assessment of aflatoxin and

fumonisin contamination in western

Kenya, 1250; effect of maize hybrid and

foliar fungicides on yield, 1080; effects of Stenocarpella macrospora on gas exchange and chlorophyll $a$ fluorescence, 26
Zebra chip disease. See 'Candidatus Liberibacter solanacearum'

Zeng, C. Y., S4.144

Zernova, O. V., S4.109

Zhang, C., S4.158

Zhang, F., S4.71, S4.84

Zhang, H., 764, 1612, S4.175

Zhang, J., S4.44, S4.157

Zhang, K., S4.157, S4.175

Zhang, M., 1458, S4.153

Zhang, N., S4.175

Zhang, P., 1458

Zhang, Q., S4.163

Zhang, R., S4.157

Zhang, S., 885, S2.8, S4.108, S4.156, S4.158

Zhang, W., 1104, 1347

Zhang, X., S4.161

Zhang, Y., 588, 1325, S4.56, S4.157, S4.158,

S4.159, S4.175

Zhang, Y. Y., S4.144

Zhang, Z., S4.163

Zhao, B., S4.40

Zhao, M., S3.1

Zhao, X., S4.147

Zhao, X. Y., S4.158

Zhao, Y., 588, S1.2, S4.33, S4.79

Zhen, F., S4.63

Zheng, J., 1458

Zheng, X., 1612

Zheng, Y., S4.32, S4.70, S4.106

Zheng, Y. L., 1206

Zheng, Z., S4.159

Zhong, S., 482

Zhou, C., S4.24

Zhou, L., S4.38

Zhou, P., S4.155

Zhou, Q., S4.26, S4.62

Zhou, T., S4.94

Zhou, X., 588, 1318, S4.157, S4.159, S4.175

Zhou, X. G., S4.140, S4.159

Zhou, Y., S4.152

Zhu, H., S4.97

Zhu, H.-Q., 220

Zhu, T., S4.144

Zhu, X. Q., 1555

Zhu, Y., S4.159

Zia-Ur-Rehman, M., S4.20, S4.159

Zidek, M., S4.160

Ziegelmann, P. K., 307

Zimmermann, B. H., S4.113

Zitnick-Anderson, K., S4.153, S4.160

Zoffoli, J. P., S4.36

Zondag, R H., S4.109

Zuchelli, E., S4.125

Zuniga, A., S4.160

Zurn, J. D., 707, S4.160

Zwart, M. P., 18 